\title{
Global existence of smooth solutions to two-dimensional compressible isentropic Euler equations for Chaplygin gases
}

\author{
De-Xing Kong ${ }^{a}$ and Yu-Zhu Wang $^{b}$ \\ ${ }^{a}$ Center of Mathematical Sciences, Zhejiang University \\ Hangzhou 310027, China \\ ${ }^{b}$ Department of Mathematics, Shanghai Jiao Tong University \\ Shanghai 200240, China
}

\begin{abstract}
In this paper we investigate the two-dimensional compressible isentropic Euler equations for Chaplygin gases. Under the assumption that the initial data is close to a constant state and the vorticity of the initial velocity vanishes, we prove the global existence of the smooth solution to the Cauchy problem for two-dimensional flow of Chaplygin gases.
\end{abstract}

Key words and phrases: Chaplygin gases, compressible Euler equations, irrotational flow, null condition, Cauchy problem, smooth solution.

2000 Mathematics Subject Classification: 76N10; 35Q35; 35L99. 


\section{Introduction}

This paper concerns the following two-dimensional compressible isentropic Euler equations

$$
\left\{\begin{array}{l}
\partial_{t} \rho+u \cdot \nabla \rho+\rho \nabla \cdot u=0, \\
\partial_{t} u+u \cdot \nabla u+\frac{1}{\rho} \nabla P=0,
\end{array}\right.
$$

where $\rho(x, t)>0$ is the density, $u(x, t)=\left(u^{1}(x, t), u^{2}(x, t)\right)^{T}$ stands for the velocity, $P$ denotes the pressure and the state equation reads

$$
P=P_{0}-A \rho^{-1}
$$

in which $P_{0}, A$ are two positive constants. We are interested in the global existence on the smooth solution of the Cauchy problem for the system (1.1) with the initial data

$$
t=0: \quad \rho=\bar{\rho}+\varepsilon \rho_{0}(x), \quad u=\varepsilon u_{0}(x),
$$

where $\varepsilon$ is a positive small parameter, $\bar{\rho}$ stands for a positive constant and satisfies

$$
P_{0}-A \bar{\rho}^{-1}>0
$$

$\rho_{0}(x)$ is a given smooth function with compact support, and $u_{0}(x)=\left(u_{0}^{1}(x), u_{0}^{2}(x)\right)^{T}$ is a given vector-valued smooth function with compact support, without loss of generality, we may assume that there exists a positive number $R$ such that

$$
\operatorname{supp}\left\{\rho_{0}\right\}, \operatorname{supp}\left\{u_{0}^{1}\right\}, \operatorname{supp}\left\{u_{0}^{2}\right\} \subset\left\{x \in \mathbb{R}^{2}|| x \mid \leq R\right\}
$$

In (1.1) and here after, we use the following notations: $x=\left(x_{1}, x_{2}\right) \in \mathbb{R}^{2}, \nabla=\left(\partial_{x_{1}}, \partial_{x_{2}}\right)$ and

$$
\partial_{0}=\partial_{t}=\frac{\partial}{\partial t}, \quad \partial_{i}=\partial_{x_{i}}=\frac{\partial}{\partial x_{i}}(i=1,2) .
$$

The compressible Euler equations comprise a nonlinear symmetric hyperbolic system of PDEs that model the ideal fluid flow. The characteristic wave speeds of this system are given by the fluid velocity and the local sound speed. The two wave families associated to these speeds may be considered separately. Smooth Eulerian flows of ideal polytropic gases with initial data close to a constant state do not, in gereral, exist for all positive times (see $[6,17,18])$. Godin [6] obtained the precise information on the asymptotic behavior of the life-span of the smooth solution to three-dimensional spherically symmetric Eulerian flows of ideal polytropic gases with variable entropy, provided that the initial data is a smooth 
small perturbation with compact support to a constant state. Sideris [17] showed that the classical solution to the three-dimensional Euler equations for a polytropic, ideal fluid must blow up in finite time under some assumption on the initial data. In Sideris [18], the estimates on life-span of smooth solutions in two space dimensions were obtained under suitable assumptions. In particular, we would like to mention that Grassin [8] proved that the suitable initial data, which force particles to spread out, may lead to the global existence in time for ideal polytropic gases.

When the adiabatic constant $\gamma$ of the gas is equal to -1 (in this case, the equation of state is suitably modified to keep the pressure and its derivative with respect to the density positive), the gas is named Chaplygin gas, or called Karman-Tsien gas (see [20, 21], and in the isentropic case, see [4]). Godin [7] obtained the global existence of a class of smooth three-dimensional spherically symmetric flows of Chaplygin gases with the variable entropy, provided that the initial data is a smooth small perturbation with compact support to a constant state.

By the light-cone gauge, Bordemann and Hoppe [2] simplified the description of relativistic membrane moving in the Minkowski space. By performing variables transformations, they established the relationship between the dynamics of relativistic membrane and two-dimensional fluid dynamics. The equations for the two-dimensional fluid dynamics derived in [2] are nothing but the system (1.1). This implies that the system (1.1) can be used to describe the motion of relativistic membrane moving in the Minkowski space $\mathbb{R}^{1+3}$. On this research topic, we refer Huang and Kong [12].

In the present paper, we investigate the global existence of the smooth solution of the two-dimensional flow of Chaplygin gases. More precisely, under the assumption that the initial data is close to a constant state and the vorticity of the initial velocity vanishes, we show the global existence of the smooth solution of the Cauchy problem (1.1)-(1.2). The main result in this paper is the following theorem.

Theorem 1.1 Suppose that the initial velocity is irrotational, i.e., $u_{0}$ satisfies

$$
\nabla^{\perp} \cdot u_{0} \triangleq \partial_{1} u_{0}^{2}-\partial_{2} u_{0}^{1}=0
$$

Then there exists $\varepsilon_{0}>0$ such that the Cauchy problem (1.1)-(1.2) has a unique global smooth solution for any $\varepsilon \in\left[0, \varepsilon_{0}\right]$.

The key idea to prove Theorem 1.1 is as follows: (i) write the velocity as the gradient of a potential function; (ii) reduce the system (1.1) to a quasilinear wave equation for 
the potential function; (iii) verify that this quasilinear wave equation satisfies the null condition in two space dimensions; (iv) by null condition, $L^{2}-L^{\infty}$ estimates and energy estimates, obtain the global existence on the smooth solution under consideration.

Remark 1.1 Theorem 1.1 still holds if the initial data $u(x, 0)=\varepsilon u_{0}(x)$ is replaced by $u(x, 0)=\bar{u}+\varepsilon u_{0}(x)$, where $\bar{u} \in \mathbb{R}^{2}$ is a constant vector. In fact, as is well known, replacing $x$ by $x-t \bar{u}$ and $u$ by $u-\bar{u}$ leads to a new solution of (1.1)-(1.2) (with the same pressure law), and so we reduce to the assumptions of Theorem 1.1 immediately.

The paper is organized as follows. In Section 2 we reduce the Cauchy problem (1.1)(1.2) to a Cauchy problem for a quasilinear wave equation (see (2.16)-(2.17) below). In Section 3, we introduce some notations and prove some lemmas, these lemmas play an important role in the proof of Theorem 1.1. Sections 4-5 are devoted to establishing the $L^{2}-L^{\infty}$ estimates and energy estimates of the smooth solution, respectively. Theorem 1.1 is proved in Section 6 by the bootstrap argument.

\section{Two-dimensional irrotational flow}

Let

$$
\nabla^{\perp}=\left(-\partial_{2}, \partial_{1}\right), \quad \omega=\left(\omega^{1}, \omega^{2}\right)
$$

then

$$
\nabla^{\perp} \cdot \omega=\partial_{1} \omega^{2}-\partial_{2} \omega^{1}
$$

As mentioned before, the key idea of the proof of Theorem 1.1 is to write the velocity $u$ as the gradient of a potential function. To do so, we first prove the following lemma which plays an important roles in the present paper.

Lemma 2.1 Let $T$ be a given positive real number and assume that $(\rho, u)$ is a smooth solution to the Cauchy problem (1.1)-(1.2) in the domain $\mathbb{R}^{2} \times[0, T)$. If the initial velocity $u_{0}(x)$ satisfies

$$
\nabla^{\perp} \cdot u_{0}=0
$$

then

$$
\nabla^{\perp} \cdot u=0, \quad \forall(x, t) \in \mathbb{R}^{2} \times[0, T)
$$


Proof. Noting $u=\left(u^{1}, u^{2}\right)$, by a direct calculation we have

$$
\begin{aligned}
\nabla^{\perp} \cdot(u \cdot \nabla u)= & \partial_{1} u^{1} \partial_{1} u^{2}+u^{1} \partial_{1}^{2} u^{2}+\partial_{1} u^{2} \partial_{2} u^{2}+u^{2} \partial_{1} \partial_{2} u^{2} \\
& -\partial_{2} u^{1} \partial_{1} u^{1}-u^{1} \partial_{2} \partial_{1} u^{1}-\partial_{2} u^{2} \partial_{2} u^{1}-u^{2} \partial_{2}^{2} u^{1} \\
(u \cdot \nabla)\left(\nabla^{\perp} \cdot u\right)= & u^{1} \partial_{1}^{2} u^{2}-u^{1} \partial_{1} \partial_{2} u^{1}+u^{2} \partial_{1} \partial_{2} u^{2}-u^{2} \partial_{2}^{2} u^{1}
\end{aligned}
$$

and

$$
(\nabla \cdot u)\left(\nabla^{\perp} \cdot u\right)=\partial_{1} u^{1} \partial_{1} u^{2}-\partial_{1} u^{1} \partial_{2} u^{1}+\partial_{2} u^{2} \partial_{1} u^{2}-\partial_{2} u^{1} \partial_{2} u^{2}
$$

$(2.3)-(2.5)$ gives

$$
\nabla^{\perp} \cdot(u \cdot \nabla u)=(u \cdot \nabla)\left(\nabla^{\perp} \cdot u\right)+(\nabla \cdot u)\left(\nabla^{\perp} \cdot u\right)
$$

Noting (2.6) and taking the curl of the second equation in (1.1) gives

$$
\partial_{t}\left(\nabla^{\perp} \cdot u\right)+u \cdot \nabla\left(\nabla^{\perp} \cdot u\right)+(\nabla \cdot u)\left(\nabla^{\perp} \cdot u\right)=0
$$

Combining (2.1) and (2.7) yields (2.2) immediately. Thus, the proof of Lemma 2.1 is completed.

Remark 2.1 Lemma 2.1 shows that, for two-dimensional compressible isentropic Euler equations for Chaplygin gases, if the flow is irrotational at the initial time, then so is it at any time $t \in[0, T)$, where $T$ stands for the maximal existence time of the smooth solution.

Under the assumptions of Theorem 1.1, for system (1.1) there exists a scalar function $v=v(x, t)$ such that

$$
u=\nabla v
$$

where the scalar function $v(x, t)$ is a smooth function and has a compact support in $x$ (since $u$ has compact support by the finite propagation speed). Substituting $u=\nabla v$ into the second equation in (1.1) and noting Lemma 2.1 leads to

$$
\partial_{t} \nabla v+\frac{1}{2} \nabla|\nabla v|^{2}+\nabla f(\rho)=0,
$$

where the function $f$ is defined by

$$
f^{\prime}(\rho)=\frac{1}{\rho} \frac{d P}{d \rho}
$$

and

$$
f(\bar{\rho})=0 .
$$


It follows from (2.10) and (2.11) that

$$
f(\rho)=\int_{\bar{\rho}}^{\rho} \frac{1}{\lambda} \frac{d P}{d \lambda} d \lambda=\frac{\bar{c}^{2}}{2}-\frac{A}{2 \rho^{2}}
$$

where

$$
\bar{c}=\left\{\frac{d P}{d \rho}(\bar{\rho})\right\}^{\frac{1}{2}}
$$

Noting (2.9), we have

$$
\partial_{t} v+\frac{1}{2}|u|^{2}+f(\rho)=0 .
$$

Thus, by (2.8) and (2.13), we obtain

$$
\left\{\begin{array}{l}
\nabla \cdot u=\Delta v \\
\partial_{t}^{2} v=-u \cdot \partial_{t} u-f^{\prime}(\rho) \partial_{t} \rho, \\
\partial_{t} \nabla v=-u \cdot \nabla u-f^{\prime}(\rho) \nabla \rho .
\end{array}\right.
$$

It follows from (2.14) that

$$
\left\{\begin{array}{l}
\nabla \cdot u=\Delta v \\
\partial_{t} \rho=-\frac{1}{f^{\prime}(\rho)}\left(\partial_{t}^{2}+\nabla v \cdot \partial_{t} \nabla v\right), \\
\nabla \rho=-\frac{1}{f^{\prime}(\rho)}\left(\nabla \partial_{t} v+\nabla v \cdot \nabla \nabla v\right) .
\end{array}\right.
$$

Substituting (2.15) into the first equation in (1.1) yields

$$
\partial_{t}^{2} v-\bar{c}^{2} \triangle v+2 \sum_{i=1}^{2} \partial_{i} v \partial_{t} \partial_{i} v-2 \partial_{t} v \triangle v+\sum_{i, j=1}^{2} \partial_{i} v \partial_{j} v \partial_{i} \partial_{j} v-|\nabla v|^{2} \triangle v=0 .
$$

On the other hand, noting (1.2), (2.8) and (2.13), we get

$$
t=0: \quad v=\varepsilon \int_{R}^{x_{1}} u_{0}^{1}\left(y, x_{2}\right) d y, \quad v_{t}=-\frac{1}{2} \varepsilon^{2}\left|u_{0}(x)\right|^{2}-f\left(\bar{\rho}+\varepsilon \rho_{0}(x)\right) .
$$

Remark 2.2 For quasilinear wave equations in two space dimensions satisfying the null condition (resp. both null conditions), Alinhac [1] proved the quasi-global existence (resp. global existence) for their Cauchy problems. His proof relies on the construction of an approximate solution, combined with an energy integral method which displays the null condition(s). The method used in the present paper is different from one in Alinhac [1].

By (2.17), it is easy to see that $v(x, 0), \partial_{t}(x, 0)$ are smooth functions of $x \in \mathbb{R}^{2}$ and has compact support, i.e.,

$$
\operatorname{supp}\{v(x, 0)\}, \operatorname{supp}\left\{\partial_{t}(x, 0)\right\} \subset\left\{x \in \mathbb{R}^{2}|| x \mid \leq R\right\} .
$$


Hence by the finite propagation speed, we obtain

$$
v(x, t)=0, \quad \forall x \in\left\{x \in \mathbb{R}^{2}|| x \mid \geq R+\bar{c} t\right\} .
$$

Obviously, (2.16) can be rewritten as

$$
\partial_{t}^{2} v-\bar{c}^{2} \triangle v+\sum_{i, j, k=0}^{2} g_{i j}^{k} \partial_{k} v \partial_{i} \partial_{j} v+\sum_{i, j, k, l=0}^{2} g_{i j}^{k l} \partial_{k} v \partial_{l} v \partial_{i} \partial_{j} v=0,
$$

where

$$
\sum_{i, j, k=0}^{2} g_{i j}^{k} \partial_{k} v \partial_{i} \partial_{j} v=2 \partial_{1} v \partial_{0} \partial_{1} v+2 \partial_{2} v \partial_{0} \partial_{2} v-2 \partial_{0} v \partial_{1}^{2} v-2 \partial_{0} v \partial_{2}^{2} v
$$

and

$$
\sum_{i, j, k, l=0}^{2} g_{i j}^{k l} \partial_{k} v \partial_{l} v \partial_{i} \partial_{j} v=2 \partial_{1} v \partial_{2} v \partial_{1} \partial_{2} v-\left(\partial_{2} v\right)^{2} \partial_{1}^{2} v-\left(\partial_{1} v\right)^{2} \partial_{2}^{2} v .
$$

For any given vector $\left(X_{0}, X_{1}, X_{2}\right) \in \mathbb{R}^{3}$ with $X_{0}^{2}=\bar{c}^{2}\left(X_{1}^{2}+X_{2}^{2}\right)$, we have

$$
\sum_{i, j, k=0}^{2} g_{i j}^{k} X_{i} X_{j} X_{k}=2 X_{0} X_{1}^{2}+2 X_{0} X_{2}^{2}-2 X_{0} X_{1}^{2}-2 X_{0} X_{2}^{2}=0
$$

and

$$
\sum_{i, j, k, l=0}^{2} g_{i j}^{k l} X_{i} X_{j} X_{k} X_{l}=2 X_{1} X_{2} X_{1} X_{2}-X_{1}^{2} X_{2}^{2}-X_{1}^{2} X_{2}^{2}=0 .
$$

This shows that the wave equation (2.16) satisfies the null condition in two space dimensions (see[1]). The concept of null condition was introduced by Christodoulou and Klainerman for nonlinear wave equations in three space dimensions (see [3] and [14]). Therefore, we can rewrite the terms

$$
\sum_{i, j, k=0}^{2} g_{i j}^{k} \partial_{k} v \partial_{i} \partial_{j} v \text { and } \sum_{i, j, k, l=0}^{2} g_{i j}^{k l} \partial_{k} v \partial_{l} v \partial_{i} \partial_{j} v
$$

as

$$
\sum_{i, j, k=0}^{2} g_{i j}^{k} \partial_{k} v \partial_{i} \partial_{j} v=2 Q_{01}\left(\partial_{1} v, v\right)+2 Q_{02}\left(\partial_{2} v, v\right)
$$

and

$$
\sum_{i, j, k, l=0}^{2} g_{i j}^{k l} \partial_{k} v \partial_{l} v \partial_{i} \partial_{j} v=-\partial_{2} v Q_{12}\left(\partial_{1} v, v\right)+\partial_{1} v Q_{12}\left(\partial_{2} v, v\right),
$$

where $Q_{i j}(\varphi, \psi)$ are defined by

$$
Q_{i j}(\varphi, \psi)=\partial_{i} \varphi \partial_{j} \psi-\partial_{i} \psi \partial_{j} \varphi \quad(0 \leq i<j \leq 2)
$$

which are null forms. 


\section{Preliminaries}

In order to prove Theorem 1.1, in this section we give some preliminaries.

Following Klainerman [13], we introduce the following vector fields

$$
\Gamma=\left(\Gamma_{0}, \cdots, \Gamma_{4}\right)=\left(\partial_{0}, \partial_{1}, \partial_{2}, \Omega, S\right)
$$

where

$$
\Omega=x_{1} \partial_{2}-x_{2} \partial_{1}, \quad S=t \partial_{t}+r \partial_{r}
$$

It is easy to show that

$$
\left\{\begin{array}{l}
{\left[\Gamma_{i}, \square\right]=-2 \delta_{4 i} \quad(i=0, \cdots, 4),} \\
{\left[\partial_{i}, \partial_{j}\right]=0(i, j=0,1,2), \quad\left[S, \partial_{i}\right]=-\partial_{i} \quad(i=0,1,2),} \\
{\left[\Omega, \partial_{1}\right]=-\partial_{2}, \quad\left[\Omega, \partial_{2}\right]=\partial_{1}, \quad\left[\Omega, \partial_{0}\right]=\partial_{0}, \quad[S, \Omega]=0}
\end{array}\right.
$$

where $[\cdot, \cdot]$ denotes the usual commutator of linear operators and $\delta_{i j}$ is the Kronecker symbol.

In what follows, we shall use the following notations

$$
\begin{gathered}
|v(x, t)|_{\kappa}=\sum_{|a| \leq \kappa}\left|\Gamma^{a} v(x, t)\right|, \quad|v(t)|_{\kappa}=\sup _{x \in \mathbb{R}^{2}}|v(x, t)|_{\kappa}, \\
{[v(x, t)]_{\kappa}=\sum_{|a| \leq \kappa}(1+|x|)^{\frac{1}{2}}(1+|x|-\bar{c} t)\left|\Gamma^{a} v(x, t)\right|, \quad[v(t)]_{\kappa}=\sup _{x \in \mathbb{R}^{2}}[v(x, t)]_{\kappa}}
\end{gathered}
$$

and

$$
\begin{gathered}
\langle v(x, t)\rangle=\sum_{|a| \leq \kappa}(1+|x|+t)^{\frac{1}{2}}\left|\Gamma^{a} v(x, t)\right|, \\
\langle v(t)\rangle=\sup _{x \in \mathbb{R}^{2}}\langle v(x, t)\rangle, \quad\|v(t)\|_{\kappa}=\sum_{|a| \leq \kappa}\left\|\Gamma^{a} v(\cdot, t)\right\|_{L^{2}},
\end{gathered}
$$

where $\kappa$ is a nonnegative integer, $a=\left(a_{0}, \cdots, a_{4}\right)$ is a multi-index and $|a|=\left|a_{0}\right|+\cdots+\left|a_{4}\right|$. Moreover, we also use the following notations

$$
|v|_{\kappa, t}=\sup _{0 \leq s<t}|v(s)|_{\kappa}, \quad[v]_{\kappa, t}=\sup _{0 \leq s<t}[v(s)]_{\kappa}
$$

and

$$
\langle v\rangle_{\kappa, t}=\sup _{0 \leq s<t}\langle v(s)\rangle_{\kappa}, \quad\|v\|_{\kappa, t}=\sup _{0 \leq s<t}\|v(s)\|_{\kappa} .
$$

Let

$$
c_{0}=\frac{1}{2} \bar{c}
$$


and define

$$
\Lambda(T)=\left\{(x, t) \in \mathbb{R}^{2} \times[0, T)|||x|-\bar{c} t \mid \leq c_{0} t\right\}
$$

Thanks to the null condition, we will be able to show that the null form possesses a good decay in the domain $\Lambda(T)$. In the our argument, the following operators

$$
Z=\left(Z_{1}, Z_{2}\right)=\frac{\bar{c} t-|x|}{t}\left(\partial_{1}, \partial_{2}\right)+\frac{\left(x_{1}, x_{2}\right) S+\left(-x_{2}, x_{1}\right) \Omega}{|x| t}
$$

will play an important role. It follows from (3.5) that

$$
Z_{i}=\bar{c} \partial_{i}+\frac{x_{i}}{|x|} \partial_{t} \quad(i=1,2)
$$

and

$$
|Z \varphi(x, t)| \leq C\left(\frac{|| x|-\bar{c} t|}{t}|\partial \varphi(x, t)|+\frac{1}{t}|\varphi(x, t)|_{1}\right),
$$

here and hereafter $C$ stands for a common absolute constant. We have

Lemma 3.1 Assume that $h_{i j}^{k}$ are constants and it holds that

$$
\sum_{i, j, k=0}^{2} h_{i j}^{k} X_{i} X_{j} X_{k}=0
$$

for any vector $\left(X_{0}, X_{1}, X_{2}\right)$ satisfying $X_{0}^{2}=\bar{c}^{2}\left(X_{1}^{2}+X_{2}^{2}\right)$. If $\varphi, \psi \in C^{\infty}\left(\mathbb{R}^{2} \times[0, T)\right)$, then

$$
\left|\sum_{i, j, k=0}^{2} h_{i j}^{k} \partial_{k} \psi \partial_{i} \partial_{j} \varphi(x, t)\right| \leq C\left[|Z \psi(x, t)|\left|\partial^{2} \varphi(x, t)\right|+|\partial \psi(x, t) \| Z \partial \varphi(x, t)|\right] .
$$

Lemma 3.2 Assume that $h_{i j}^{k l}$ are constants and it holds that

$$
\sum_{i, j, k, l=0}^{2} h_{i j}^{k l} X_{i} X_{j} X_{k} X_{l}=0
$$

for any vector $\left(X_{0}, X_{1}, X_{2}\right)$ satisfying $X_{0}^{2}=\bar{c}^{2}\left(X_{1}^{2}+X_{2}^{2}\right)$. If $\varphi, \psi, \phi \in C^{\infty}\left(\mathbb{R}^{2} \times[0, T)\right)$, then

$$
\begin{aligned}
& \left|\sum_{i, j, k, l=0}^{2} h_{i j}^{k l} \partial_{k} \psi \partial_{l} \phi \partial_{i} \partial_{j} \varphi(x, t)\right| \\
& \leq C\left[|\partial \psi(x, t)\|\partial \phi(x, t)\| Z \partial \varphi(x, t)|+|\partial \psi(x, t)|\left|Z \phi(x, t)\left\|\partial^{2} \varphi(x, t)|+| Z \psi(x, t)\right\| \partial \phi(x, t) \| \partial^{2} \varphi(x, t)\right|\right] .
\end{aligned}
$$

In what follows, we only prove Lemma 3.2 , because the proof of Lemma 3.1 is of the same type and even somewhat simpler. 
Proof. In fact, it follows from (3.10) that

$$
\begin{aligned}
\sum_{i, j, k, l=0}^{2} h_{i j}^{k l} \partial_{k} \psi \partial_{l} \phi \partial_{i} \partial_{j} \varphi=\sum_{i, j, k, l=0}^{2} \frac{1}{\bar{c}} h_{i j}^{k l}\left(\bar{c} \partial_{k}+\omega_{k} \partial_{t}\right) \psi \partial_{l} \phi \partial_{i} \partial_{j} \varphi \\
\quad-\sum_{i, j, k, l=0}^{2} h_{i j}^{k l} \frac{\omega_{k}}{\bar{c}^{2}} \partial_{k} \psi\left(\bar{c} \partial_{l}+\omega_{l} \partial_{t}\right) \phi \partial_{i} \partial_{j} \varphi+\sum_{i, j, k, l=0}^{2} h_{i j}^{k l} \frac{\omega_{k} \omega_{l}}{\bar{c}^{3}} \partial_{k} \psi \partial_{l} \phi\left(\bar{c} \partial_{i}+\omega_{i} \partial_{t}\right) \partial_{j} \varphi \\
\quad-\sum_{i, j, k, l=0}^{2} h_{i j}^{k l} \frac{\omega_{k} \omega_{l} \omega_{i}}{\bar{c}^{4}} \partial_{k} \psi \partial_{l} \phi\left(\bar{c} \partial_{j}+\omega_{j} \partial_{t}\right) \partial_{t} \varphi+\sum_{i, j, k, l=0}^{2} h_{i j}^{k l} \frac{\omega_{k} \omega_{l} \omega_{i} \omega_{j}}{\bar{c}^{4}} \partial_{t} \psi \partial_{t} \phi \partial_{t}^{2} \varphi \\
=\sum_{i, j, k, l=0}^{2} h_{i j}^{k l} \frac{1}{\bar{c}}\left(\bar{c} \partial_{k}+\omega_{k} \partial_{t}\right) \psi \partial_{l} \phi \partial_{i} \partial_{j} \varphi-\sum_{i, j, k, l=0}^{2} h_{i j}^{k l} \frac{\omega_{k}}{\bar{c}^{2}} \partial_{k} \psi\left(\bar{c} \partial_{l}+\omega_{l} \partial_{t}\right) \phi \partial_{i} \partial_{j} \varphi \\
\quad+\sum_{i, j, k, l=0}^{2} h_{i j}^{k l} \frac{\omega_{k} \omega_{l}}{\bar{c}^{3}} \partial_{k} \psi \partial_{l} \phi\left(\bar{c} \partial_{i}+\omega_{i} \partial_{t}\right) \partial_{j} \varphi-\sum_{i, j, k, l=0}^{2} h_{i j}^{k l} \frac{\omega_{k} \omega_{l} \omega_{i}}{\bar{c}^{4}} \partial_{k} \psi \partial_{l} \phi\left(\bar{c} \partial_{j}+\omega_{j} \partial_{t}\right) \partial_{t} \varphi
\end{aligned}
$$

where

$$
\left(\omega_{0}, \omega_{1}, \omega_{2}\right)=\left(-\bar{c}, \frac{x_{1}}{|x|}, \frac{x_{2}}{|x|}\right) .
$$

Noting (3.6) and (3.12), we obtain (3.11) immediately. This completes the proof of Lemma 3.2 .

Lemma 3.3 There exist constants $\tilde{g}_{i j}^{k}$ such that (i) $\sum_{i, j, k=0}^{2} \tilde{g}_{i j}^{k} \partial_{k} v \partial_{i} \partial_{j} v$ satisfies the null condition, that is, for any vector $\left(X_{0}, X_{1}, X_{2}\right)$ satisfying $X_{0}^{2}=\bar{c}^{2}\left(X_{1}^{2}+X_{2}^{2}\right)$, it holds that

$$
\sum_{i, j, k=0}^{2} \tilde{g}_{i j}^{k} X_{i} X_{j} X_{k}=0
$$

(ii) the following equality holds

$$
\Gamma\left(\sum_{i, j, k=0}^{2} g_{i j}^{k} \partial_{k} v \partial_{i} \partial_{j} v\right)=\sum_{i, j, k=0}^{2} g_{i j}^{k} \partial_{k} \Gamma v \partial_{i} \partial_{j} v+\sum_{i, j, k=0}^{2} g_{i j}^{k} \partial_{k} v \partial_{i} \partial_{j} \Gamma v+\sum_{i, j, k=0}^{2} \tilde{g}_{i j}^{k} \partial_{k} v \partial_{i} \partial_{j} v .
$$

Lemma 3.4 There exist constants $\tilde{g}_{i j}^{k l}$ such that (I) $\sum_{i, j, k, l=0}^{2} \tilde{g}_{i j}^{k l} \partial_{k} v \partial_{l} v \partial_{i} \partial_{j} v$ satisfies the null condition, that is, for any vector $\left(X_{0}, X_{1}, X_{2}\right)$ satisfying $X_{0}^{2}=\bar{c}^{2}\left(X_{1}^{2}+X_{2}^{2}\right)$, it holds that

$$
\sum_{i, j, k, l=0}^{2} \tilde{g}_{i j}^{k l} X_{i} X_{j} X_{k} X_{j}=0
$$


(II) the following equality holds

$$
\begin{aligned}
\Gamma\left(\sum_{i, j, k, l=0}^{2} g_{i j}^{k l} \partial_{k} v \partial_{l} v \partial_{i} \partial_{j} v\right)= & \sum_{i, j, k, l=0}^{2} g_{i j}^{k l} \partial_{k} \Gamma v \partial_{l} v \partial_{i} \partial_{j} v+\sum_{i, j, k, l=0}^{2} g_{i j}^{k l} \partial_{k} v \partial_{l} \Gamma v \partial_{i} \partial_{j} v \\
& +\sum_{i, j, k, l=0}^{2} g_{i j}^{k l} \partial_{k} v \partial_{l} \partial_{i} \partial_{j} \Gamma v+\sum_{i, j, k, l=0}^{2} \tilde{g}_{i j}^{k l} \partial_{k} v \partial_{l} v \partial_{i} \partial_{j} v .
\end{aligned}
$$

As before, in what follows we only prove Lemma 3.4, because the proof of Lemma 3.3 is of the same type and even somewhat simpler.

Proof. It follows from (3.3) that

$$
\begin{gathered}
\partial_{m}\left(\sum_{i, j, k, l=0}^{2} g_{i j}^{k l} \partial_{k} v \partial_{l} v \partial_{i} \partial_{j} v\right)=\sum_{i, j, k, l=0}^{2} g_{i j}^{k l} \partial_{k} \partial_{m} v \partial_{l} v \partial_{i} \partial_{j} v+ \\
\sum_{i, j, k, l=0}^{2} g_{i j}^{k l} \partial_{k} v \partial_{l} \partial_{m} v \partial_{i} \partial_{j} v+\sum_{i, j, k, l=0}^{2} g_{i j}^{k l} \partial_{k} v \partial_{l} v \partial_{i} \partial_{j} \partial_{m} v \\
S\left(\sum_{i, j, k, l=0}^{2} g_{i j}^{k l} \partial_{k} v \partial_{l} v \partial_{i} \partial_{j} v\right)=\sum_{i, j, k, l=0}^{2} g_{i j}^{k l} \partial_{k} S v \partial_{l} v \partial_{i} \partial_{j} v+\sum_{i, j, k, l=0}^{2} g_{i j}^{k l} \partial_{k} v \partial_{l} S v \partial_{i} \partial_{j} v+ \\
\sum_{i, j, k, l=0}^{2} g_{i j}^{k l} \partial_{k} v \partial_{l} \partial_{i} \partial_{j} S v-4 \sum_{i, j, k, l=0}^{2} g_{i j}^{k l} \partial_{k} v \partial_{l} v \partial_{i} \partial_{j} v
\end{gathered}
$$

and

$$
\begin{array}{r}
\Omega\left(\sum_{i, j, k, l=0}^{2} g_{i j}^{k l} \partial_{k} v \partial_{l} v \partial_{i} \partial_{j} v\right)=2 \partial_{1} \Omega v \partial_{2} v \partial_{1} \partial_{2} v+2 \partial_{1} v \partial_{2} \Omega v \partial_{1} \partial_{2} v+2 \partial_{1} v \partial_{2} v \partial_{1} \partial_{2} \Omega v- \\
2 \partial_{1} \Omega v \partial_{1} v \partial_{2}^{2} v-\left(\partial_{1} v\right)^{2} \partial_{2}^{2} \Omega v-2 \partial_{2} v \partial_{2} \Omega v \partial_{1}^{2} v-\left(\partial_{2} v\right)^{2} \partial_{1}^{2} \Omega v \\
=\sum_{i, j, k, l=0}^{2} g_{i j}^{k l} \partial_{k} \Omega v \partial_{l} v \partial_{i} \partial_{j} v+\sum_{i, j, k, l=0}^{2} g_{i j}^{k l} \partial_{k} v \partial_{l} \Omega v \partial_{i} \partial_{j} v+\sum_{i, j, k, l=0}^{2} g_{i j}^{k l} \partial_{k} v \partial_{l} \partial_{i} \partial_{j} \Omega v .
\end{array}
$$

The desired (3.15)-(3.16) follows from (3.17)-(3.19) immediately. This proves Lemma 3.4.

By Lemmas 3.1-3.4, we can obtain the following lemma.

Lemma 3.5 Let $v=v(x, t)$ be a smooth solution of the Cauchy problem (2.16)-(2.17) on the domain $\mathbb{R}^{2} \times[0, T)$ and $\kappa$ be a positive integer, where $T$ is a given positive real number. 
Then there exists a positive constant $C_{\kappa}$ depending on $\kappa$ but independent of $T$ such that the following inequalities hold for every $(x, t) \in \Lambda(T) \bigcap\{(y, s) \mid s \geq 1\}$

$$
\begin{array}{r}
\left|\sum_{i, j, k=0}^{2} g_{i j}^{k} \partial_{k} v \partial_{i} \partial_{j} v(x, t)\right| \leq C_{\kappa} \sum_{|a+b| \leq \kappa+1}\left|Z \Gamma^{a} v(x, t)\right|\left|\Gamma^{b} \partial v(x, t)\right|, \\
\sum_{|a| \leq \kappa}\left|\Gamma^{a}\left(\sum_{i, j, k=0}^{2} g_{i j}^{k} \partial_{k} v \partial_{i} \partial_{j} v\right)(x, t)-\sum_{i, j, k=0}^{2} g_{i j}^{k} \partial_{k} v \partial_{i} \partial_{j} \Gamma^{a} v(x, t)\right| \\
\leq C_{\kappa} \sum_{|b+c| \leq \kappa+1,|b|,|c| \leq \kappa}\left|Z \Gamma^{b} v(x, t)\right|\left|\Gamma^{c} \partial v(x, t)\right|
\end{array}
$$

and

$$
\left|\sum_{i, j, k, l=0}^{2} g_{i j}^{k l} \partial_{k} v \partial_{l} v \partial_{i} \partial_{j} v(x, t)\right|_{\kappa} \leq C_{\kappa} \sum_{|a+b+c| \leq \kappa+1}\left|Z \Gamma^{a} v(x, t)\right|\left|\Gamma^{b} \partial v(x, t)\right|\left|\Gamma^{c} \partial v(x, t)\right|,
$$

where the terms

$$
\sum_{i, j, k=0}^{2} g_{i j}^{k} \partial_{k} v \partial_{i} \partial_{j} v \text { and } \sum_{i, j, k, l=0}^{2} g_{i j}^{k l} \partial_{k} v \partial_{l} v \partial_{i} \partial_{j} v
$$

are defined by (2.20) and (2.21), respectively. Moreover, it holds that

$$
\begin{gathered}
\left|\sum_{i, j, k=0}^{2} g_{i j}^{k} \partial_{k} v \partial_{i} \partial_{j} v(x, t)\right|_{\kappa} \leq C_{\kappa} \frac{|| x|-\bar{c} t|}{1+|x|+t}|\partial v(x, t)|_{\left[\frac{\kappa+1}{2}\right]}|\partial v(x, t)|_{\kappa+1}+ \\
C_{\kappa} \frac{1}{1+|x|+t}\left(|v(x, t)|_{\left[\frac{\kappa+1}{2}\right]+1}|\partial v(x, t)|_{\kappa+1}+|v(x, t)|_{\left[\frac{\kappa+1}{2}\right]}|v(x, t)|_{\kappa+2}\right), \\
\left|\sum_{i, j, k, l=0}^{2} g_{i j}^{k l} \partial_{k} v \partial_{l} v \partial_{i} \partial_{j} v(x, t)\right|_{\kappa} \leq C_{\kappa} \frac{|| x|-\bar{c} t|}{1+|x|+t}|\partial v(x, t)|_{\left[\frac{\kappa+1}{2}\right]}^{2}|\partial v(x, t)|_{\kappa+1}+ \\
C_{\kappa} \frac{1}{1+|x|+t}|\partial v(x, t)|_{\left[\frac{\kappa+1}{2}\right]}\left(|v(x, t)|_{\left[\frac{\kappa+1}{2}\right]+1}|\partial v(x, t)|_{\kappa+1}+|v(x, t)|_{\left[\frac{\kappa+1}{2}\right]}|v(x, t)|_{\kappa+2}\right)
\end{gathered}
$$

and

$$
\begin{aligned}
\sum_{|a| \leq \kappa} \mid \Gamma^{a} & \left(\sum_{i, j, k=0}^{2} g_{i j}^{k} \partial_{k} v \partial_{i} \partial_{j} v\right)(x, t)-\sum_{i, j, k=0}^{2} g_{i j}^{k} \partial_{k} v \partial_{i} \partial_{j} \Gamma^{a} v(x, t) \mid \\
\leq & C_{\kappa} \frac{|| x|-\bar{c} t|}{1+|x|+t}|\partial v(x, t)|_{\left[\frac{\kappa+1}{2}\right]}|\partial v(x, t)|_{\kappa}+ \\
& C_{\kappa} \frac{1}{1+|x|+t}\left(|v(x, t)|_{\left[\frac{\kappa+1}{2}\right]+1}|\partial v(x, t)|_{\kappa}+|v(x, t)|_{\left[\frac{\kappa+1}{2}\right]}|v(x, t)|_{\kappa+1}\right) .
\end{aligned}
$$


Proof. We only prove (3.20), (3.21), (3.23) and (3.25), because the proof of (3.22) and (3.24) is similar.

By Lemma 3.3, we obtain

$$
\Gamma^{a}\left(\sum_{i, j, k=0}^{2} g_{i j}^{k} \partial_{k} v \partial_{i} \partial_{j} v\right)=\sum_{b+c \leq a} \sum_{i, j, k=0}^{2} g_{i j b c}^{k a} \partial_{k} \Gamma^{b} v \partial_{i} \partial_{j} \Gamma^{c} v
$$

where $\sum_{i, j, k=0}^{2} g_{i j b c}^{k a} \partial_{k} \Gamma^{b} v \partial_{i} \partial_{j} \Gamma^{c} v$ satisfies the null condition, that is, for any vector $\left(X_{0}, X_{1}, X_{2}\right)$ satisfying $X_{0}^{2}=\bar{c}^{2}\left(X_{1}^{2}+X_{2}^{2}\right)$, it holds that

$$
\sum_{i, j, k=0}^{2} g_{i j b c}^{k a} X_{i} X_{j} X_{k}=0
$$

Noting (3.3), (3.9) and (3.26), we get (3.20) immediately.

By Lemma 3.3 again, we have

$$
\Gamma^{a}\left(\sum_{i, j, k=0}^{2} g_{i j}^{k} \partial_{k} v \partial_{i} \partial_{j} v\right)-\sum_{i, j, k=0}^{2} g_{i j}^{k} \partial_{k} v \partial_{i} \partial_{j} \Gamma^{a} v=\sum_{b+c \leq a, c<a} \sum_{i, j, k=0}^{2} g_{i j b c}^{k a} \partial_{k} \Gamma^{b} v \partial_{i} \partial_{j} \Gamma^{c} v
$$

where $\sum_{i, j, k=0}^{2} g_{i j b c}^{k a} \partial_{k} \Gamma^{b} v \partial_{i} \partial_{j} \Gamma^{c} v$ satisfies the null condition, that is, for any vector $\left(X_{0}, X_{1}, X_{2}\right)$ satisfying $X_{0}^{2}=\bar{c}^{2}\left(X_{1}^{2}+X_{2}^{2}\right)$, it holds that

$$
\sum_{i, j, k=0}^{2} g_{i j b c}^{k a} X_{i} X_{j} X_{k}=0 .
$$

Thus, using (3.3), (3.9) and (3.27), we obtain (3.21).

Finally, noting (3.7), (3.20) and using (3.21), we get (3.23) and (3.25) immediately. Thus, the proof of Lemma 3.5 is completed.

\section{$4 \quad L^{2}-L^{\infty}$ estimates}

The $L^{2}-L^{\infty}$ estimates play an important role in the proof of Theorem 1.1. This section is devoted to establishing these estimates.

Theorem 4.1 Let $v=v(x, t)$ be a smooth solution of the Cauchy problem (2.16)-(2.17) on the domain $\mathbb{R}^{2} \times[0, T)$, where $T$ is a given positive real number, and let $\kappa$ be a positive integer, and $\delta$ be a positive small constant. Suppose that

$$
[\partial v]_{\left[\frac{\kappa+6}{2}\right], T}+\langle v\rangle_{\left[\frac{\kappa+6}{2}\right], T} \leq \delta
$$


then there exist positive constants $C_{0}, C_{1}$ and $\nu$ such that it holds that

$$
[\partial v(t)]_{\kappa}+\langle v(t)\rangle_{\kappa+1} \leq C_{0} \varepsilon+C_{1}\left([\partial v]_{\left[\frac{\kappa+6}{2}\right], t}+\langle v\rangle_{\left[\frac{\kappa+6}{2}\right], t}\right) \sup _{0 \leq \tau<t}\left\{(1+\tau)^{-\nu}\|\partial v(\tau)\|_{\kappa+8}\right\}
$$

for every $t \in[0, T)$.

Proof. For $t \in[0,1]$, we have

$$
\left|\partial_{t} v(x, t)\right|_{\kappa} \leq C|v(x, t)|_{\kappa+1} \leq \frac{C}{1+t}|v(x, t)|_{\kappa+1} .
$$

On the other hand, noting the fact that

$$
\partial_{t}=\frac{S}{t}-\frac{x \cdot \nabla}{t}
$$

and using (2.18), we obtain

$$
\left|\partial_{t} v(x, t)\right|_{\kappa} \leq C|\nabla v(x, t)|_{\kappa}+\frac{C}{1+t}|v(x, t)|_{\kappa+1} \quad \text { for } t \geq 1 .
$$

Thus, it follows from (4.3) and (4.4) that, for every $t \in[0, T)$

$$
\left|\partial_{t} v(x, t)\right|_{\kappa} \leq C|\nabla v(x, t)|_{\kappa}+\frac{C}{1+t}|v(x, t)|_{\kappa+1} .
$$

Thanks to (4.5), in what follows, we only need to prove (4.2) with $\partial$ replaced by $\partial_{i}(i=1,2)$ in the left-hand side. To do so, we can rewrite the solution $v=v(x, t)$ of the Cauchy problem $(2.16)-(2.17)$ as

$$
v=v_{1}+\Phi(F),
$$

where $v_{1}$ is the solution of the following Cauchy problem

$$
\left\{\begin{array}{l}
\partial_{t}^{2} v_{1}-\bar{c}^{2} \triangle v_{1}=0 \\
t=0: \quad v_{1}=\varepsilon \int_{M}^{x_{1}} u_{0}^{1}\left(y, x_{2}\right) d y, \quad \partial_{t} v_{1}=-\frac{1}{2} \varepsilon^{2}\left|u_{0}(x)\right|^{2}-f\left(\bar{\rho}+\varepsilon \rho_{0}(x)\right),
\end{array}\right.
$$

while $\Phi(F)$ is the solution of the following Cauchy problem

$$
\left\{\begin{array}{l}
\partial_{t}^{2} \Phi(F)-\bar{c}^{2} \triangle \Phi(F)=F \\
t=0: \quad \Phi(F)=0, \quad \partial_{t} \Phi(F)=0
\end{array}\right.
$$

in which

$$
F=-\sum_{i, j, k=0}^{2} g_{i j}^{k} \partial_{k} v \partial_{i} \partial_{j} v-\sum_{i, j, k, l=0}^{2} g_{i j}^{k l} \partial_{k} v \partial_{l} v \partial_{i} \partial_{j} v .
$$

On the other hand, it follows from (4.6) that (see [5] and [16])

$$
\left[\partial v_{1}(t)\right]_{\kappa}+\left\langle v_{1}(t)\right\rangle_{\kappa+1} \leq C \varepsilon \text { for } t \geq 0 .
$$


In order to prove (4.2), it suffices to show

$$
\begin{aligned}
& {[\nabla \Phi(F)(t)]_{\kappa}+\langle\Phi(F)(t)\rangle_{\kappa+1} \leq} \\
& C \varepsilon+C_{1}\left([\partial v]_{\left[\frac{\kappa+6}{2}\right], t}+\langle v\rangle_{\left[\frac{\kappa+6}{2}\right], t}\right) \sup _{0 \leq \tau<t}\left\{(1+\tau)^{-\nu}\|\partial v(\tau)\|_{\kappa+8}\right\}
\end{aligned}
$$

for $t \in[0, T)$. Here and hereafter, we interpret $[\nabla \Phi(F)(t)]_{\kappa}$ and $\langle\Phi(F)(t)\rangle_{\kappa+1}$ as

$$
[\nabla \Phi(F)(t)]_{\kappa}=\sup _{x \in \mathbb{R}^{2}} \sum_{|a| \leq \kappa}(1+|x|)^{\frac{1}{2}}(1+|x|-\bar{c} t)\left|\Gamma^{a} \nabla \Phi(F)(x, t)\right|
$$

and

$$
\langle\Phi(F)(t)\rangle_{\kappa}=\sup _{x \in \mathbb{R}^{2}} \sum_{|a| \leq \kappa}(1+|x|+t)^{\frac{1}{2}}\left|\Gamma^{a} \Phi(F)(x, t)\right| .
$$

To prove (4.10), we need the following lemmas.

Lemma 4.1 Suppose that $F \in C^{\infty}\left(\mathbb{R}^{2} \times[0, T)\right)$. Then for any given positive real number $\mu>0$ there exists a positive constant $C=C(\mu)$ such that the following inequalities holds

$$
\langle\Phi(F)(x, t)\rangle_{k} \leq C L_{\mu, \kappa}(F)(x, t)
$$

and

$$
[\nabla \Phi(F)(x, t)]_{k} \leq C L_{\mu, \kappa+1}(F)(x, t)
$$

where

$$
\begin{aligned}
L_{\mu, \kappa}(F)(x, t)= & \sup _{(y, \tau) \in D(x, t) \cap \Lambda(t)}\left\{|y|^{\frac{1}{2}}(1+|y|+\tau)^{1+\mu}(1+|| y|-\bar{c} \tau|)|F(y, \tau)|_{\kappa}\right\}+ \\
& \sup _{(y, \tau) \in D(x, t) \cap \Lambda(t)^{c}}\left\{|y|^{\frac{1}{2}}(1+|y|+\tau)^{1+\mu}(1+|y|)|F(y, \tau)|_{\kappa}\right\},
\end{aligned}
$$

in which

$$
D(x, t)=\left\{(y, \tau) \in \mathbb{R}^{2} \times[0, t]|| y-x \mid \leq \bar{c}(t-\tau)\right\} .
$$

and $\Lambda(t)^{c}$ denotes the complementary set of $\Lambda(t)$.

Lemma 4.1 can be found in [10] (see also [11]). The following Lemma comes from [15].

Lemma 4.2 Suppose that $h \in C^{2}\left(\mathbb{R}^{2} \times[0, T)\right)$ and satisfies $\|h(t)\|_{2}<\infty$. Then there exits a positive constant $C_{*}$ independent of $T$ such that the following estimate holds:

$$
|x|^{\frac{1}{2}}|h(x, t)| \leq C_{*}\|h(t)\|_{2}, \quad \forall t \in[0, T) .
$$


In what follows, we prove a more general lemma (see Lemma 4.3 below), whose special case is nothing but (4.10) (more precisely, the case $\theta=0$ in Lemma 4.3 gives (4.10)). After the proof of Theorem 4.1, we shall prove Lemma 4.3.

Lemma 4.3 Let $v=v(x, t)$ be the smooth solution of the Cauchy problem (2.16)-(2.17) on the domain $\mathbb{R}^{2} \times[0, T)$ ). Suppose that the following estimate holds

$$
[\partial v]_{\left[\frac{\kappa+6}{2}\right]-\theta, T}+\langle v\rangle_{\left[\frac{\kappa+6}{2}\right]-\theta, T} \leq \delta
$$

where the positive constants $\kappa$ and $\delta$ are defined in Theorem 4.1. Then there exists positive constant $\nu$ such that for any $t \in[0, T)$, it holds that

$$
\begin{aligned}
& (1+|x|+t)^{\left(-\frac{1}{2}+\mu\right) \theta}\left([\nabla \Phi(F)(x, t)]_{\kappa}+\langle\Phi(F)(x, t)\rangle_{\kappa+1}\right) \\
& \quad \leq C\left([\partial v]_{\left[\frac{\kappa+6}{2}\right]-\theta, t}+\left(1-\delta_{2 \theta}\right)\langle v\rangle_{\left[\frac{\kappa+6}{2}\right]-\theta, t}\right)\left(\varepsilon+\sup _{0 \leq \tau<t}\left\{(1+\tau)^{-\nu}\|\partial v(\tau)\|_{\kappa+8-2 \theta}\right\}\right)
\end{aligned}
$$

where $\mu$ is a positive constant satisfying $\mu \in\left(0, \frac{1}{6}\right), \theta$ takes its value in $\{0,1,2\}$ and $\delta_{2 \theta}$ is the Kronecker symbol.

We will prove Lemma 4.3 after the proof of Theorem 4.1.

Proof of Theorem 4.1. Obviously, taking $\theta=0$ in (4.17) gives the desired (4.10) immediately. This proves Theorem 4.1.

We now prove Lemma 4.3.

Proof of Lemma 4.3. We distinguish three cases.

Case 1: $\theta=2$

In this case, it follows from (4.15) and (4.16) that, for every $(y, \tau) \in D(x, t) \bigcap \Lambda(t)$ it holds that

$$
\begin{aligned}
|y|^{\frac{1}{2}} & (1+|y|+\tau)^{3 \mu}(1+|| y|-\bar{c} \tau|)|F(y, \tau)|_{\kappa+1} \\
& \leq C|y|^{\frac{1}{2}}(1+|y|+\tau)^{3 \mu}(1+|| y|-\bar{c} \tau|)|\partial v(y, \tau)|_{\left[\frac{\kappa+2}{2}\right]}|\partial v(y, \tau)|_{\kappa+2} \\
& \leq C|y|^{\frac{1}{2}}(1+|y|+\tau)^{3 \mu}(1+|y|)^{-\frac{1}{2}}(1+|y|)^{\frac{1}{2}}(1+|| y|-\bar{c} \tau|)|\partial v(y, \tau)|_{\left[\frac{\kappa+2}{2}\right]}|\partial v(y, \tau)|_{\kappa+2} \\
& \leq C[\partial v]_{\left[\frac{\kappa+2}{2}\right], t} \sup _{0 \leq \tau<t}\left\{(1+\tau)^{-\frac{1}{2}+3 \mu}\|\partial v(\tau)\|_{\kappa+4}\right\} .
\end{aligned}
$$


Similarly, we obtain from (2.18), (4.15) and (4.16) that

$$
\begin{aligned}
|y|^{\frac{1}{2}}(1+\mid & y \mid+\tau)^{3 \mu}(1+|y|)|F(y, \tau)|_{\kappa+1} \\
& \leq C|y|^{\frac{1}{2}}(1+|y|+\tau)^{3 \mu}(1+|y|)|\partial v(y, \tau)|_{\left[\frac{\kappa+2}{2}\right]}|\partial v(y, \tau)|_{\kappa+2} \\
& \leq C[\partial v]_{\left[\frac{\kappa+2}{2}\right]} \sup _{0 \leq \tau<t}\left\{(1+\tau)^{-\frac{1}{2}+3 \mu}\|\partial v(\tau)\|_{\kappa+4}\right\}
\end{aligned}
$$

for $(y, \tau) \in D(x, t) \bigcap \Lambda(t)^{c}$. On the other hand, by (4.11) and (4.12) and the fact that

$$
1+|y|+\tau \leq C(1+|x|+t), \quad \forall(y, \tau) \in D(x, t),
$$

we have

$$
\begin{aligned}
(1+|x|+ & t)^{-1+2 \mu}\left\{[\nabla \Phi(F)(x, t)]_{\kappa}+\langle\Phi(F)(x, t)\rangle_{\kappa+1}\right\} \\
& \leq C\left\{\sup _{(y, \tau) \in D(x, t) \cap \Lambda(t)}\left\{|y|^{\frac{1}{2}}(1+|y|+\tau)^{3 \mu}(1+|| y|-\bar{c} \tau|)|F(y, \tau)|_{\kappa+1}\right\}\right. \\
& \left.+\sup _{(y, \tau) \in D(x, t) \cap \Lambda(t)^{c}}\left\{|y|^{\frac{1}{2}}(1+|y|+\tau)^{3 \mu}(1+|y|)|F(y, \tau)|_{\kappa+1}\right\}\right\} .
\end{aligned}
$$

Combining (4.18)-(4.19), (4.21) and choosing $\nu \in\left(0, \frac{1}{2}-3 \mu\right)$ gives (4.17) in the case $\theta=2$.

Case 2: $\theta=1$

In the present situation, it follows from (4.8), (4.11), (4.12) and (4.20) that

$$
\begin{aligned}
& (1+|x|+t)^{-\frac{1}{2}+\mu}\left([\nabla \Phi(F)(x, t)]_{\kappa}+\langle\Phi(F)(x, t)\rangle_{\kappa+1}\right) \\
\leq & C\left\{\sup _{(y, \tau) \in D(x, t) \cap \Lambda(t)} A(y, \tau)+\sup _{(y, \tau) \in D(x, t) \cap \Lambda(t)^{c}} B(y, \tau)\right\},
\end{aligned}
$$

where

$$
\begin{gathered}
A(y, \tau)=|y|^{\frac{1}{2}}(1+|y|+\tau)^{\frac{1}{2}+2 \mu}(1+|| y|-\bar{c} \tau|) \\
\times\left(\left|\sum_{i, j, k=0}^{2} g_{i j}^{k} \partial_{k} v \partial_{i} \partial_{j} v(y, \tau)\right|_{\kappa+1}+\left|\sum_{i, j, k, l=0}^{2} g_{i j}^{k l} \partial_{k} v \partial_{l} v \partial_{i} \partial_{j} v(y, \tau)\right|_{\kappa+1}\right)
\end{gathered}
$$

and

$$
B(y, \tau)=|y|^{\frac{1}{2}}(1+|y|+\tau)^{\frac{1}{2}+2 \mu}(1+|y|)\left(\left|\sum_{i, j, k=0}^{2} g_{i j}^{k} \partial_{k} v \partial_{i} \partial_{j} v(y, \tau)\right|_{\kappa+1}+\left|\sum_{i, j, k, l=0}^{2} g_{i j}^{k l} \partial_{k} v \partial_{l} v \partial_{i} \partial_{j} v(y, \tau)\right|_{\kappa+1}\right) .
$$

When $\tau \in[0,1]$, it follows from (4.15) and (4.16) that, for every $(y, \tau) \in D(x, t) \bigcap \Lambda(t)$

$$
\begin{gathered}
(1+|y|+\tau)^{\frac{1}{2}+2 \mu}(1+|| y|-\bar{c} \tau|)|y|^{\frac{1}{2}}\left(\left|\sum_{i, j, k=0}^{2} g_{i j}^{k} \partial_{k} v \partial_{i} \partial_{j} v(y, \tau)\right|_{\kappa+1}+\left|\sum_{i, j, k, l=0}^{2} g_{i j}^{k l} \partial_{k} v \partial_{l} v \partial_{i} \partial_{j} v(y, \tau)\right|_{\kappa+1}\right) \\
\leq C[\partial v]_{\left[\frac{\kappa+2}{2}\right], t}\|\partial v(\tau)\|_{\kappa+4} \leq C[\partial v]_{\left[\frac{\kappa+2}{2}\right], t}(1+\tau)^{-\nu}\|\partial v(\tau)\|_{\kappa+4}, \quad \forall \nu \in \mathbb{R} .
\end{gathered}
$$


Similarly, when $\tau \in[0,1]$, it follows from (2.18), (4.15) and (4.16) that, for every $(y, \tau) \in$ $D(x, t) \bigcap \Lambda(t)^{c}$

$$
\begin{aligned}
& (1+|y|)(1+|y|+\tau)^{\frac{1}{2}+2 \mu}|y|^{\frac{1}{2}}\left(\left|\sum_{i, j, k=0}^{2} g_{i j}^{k} \partial_{k} v \partial_{i} \partial_{j} v(y, \tau)\right|_{\kappa+1}+\left|\sum_{i, j, k, l=0}^{2} g_{i j}^{k l} \partial_{k} v \partial_{l} v \partial_{i} \partial_{j} v(y, \tau)\right|_{\kappa+1}\right) \\
& \leq C[\partial v]_{\left[\frac{\kappa+2}{2}\right], t}(1+\tau)^{-\nu}\|\partial v(\tau)\|_{\kappa+4}, \quad \forall \nu \in \mathbb{R} .
\end{aligned}
$$

Thus, in what follows, it suffices to investigate the case $\tau \geq 1$.

By (3.23), (4.5), (4.9) and (4.17) with $\theta=2$, for $(y, \tau) \in D(x, t) \bigcap \Lambda(t) \bigcap\{(z, s) \mid s \geq 1\}$ we have

$$
\begin{aligned}
& (1+|y|+\tau)^{\frac{1}{2}+2 \mu}|y|^{\frac{1}{2}}(1+|| y|-\bar{c} \tau|)\left|\sum_{i, j, k=0}^{2} g_{i j}^{k} \partial_{k} v \partial_{i} \partial_{j} v(y, \tau)\right|_{\kappa+1} \\
& \leq C|y|^{\frac{1}{2}}(1+|y|+\tau)^{-\frac{1}{2}+2 \mu}\left\{(1+|| y|-\bar{c} \tau|)^{2}|\partial v(y, \tau)|_{\left[\frac{\kappa+2}{2}\right]}|\partial v(y, \tau)|_{\kappa+2}+\right. \\
& \left.(1+|| y|-\bar{c} \tau|)\left(|\partial v(y, \tau)|_{\left[\frac{\kappa+2}{2}\right]}|v(y, \tau)|_{\kappa+3}+|v(y, \tau)|_{\left[\frac{\kappa+4}{2}\right]}|\partial v(y, \tau)|_{\kappa+2}\right)\right\} \\
& \leq C\left([\partial v]_{\left[\frac{\kappa+2}{2}\right], t}+\langle v\rangle_{\left[\frac{\kappa+4}{2}\right], t}\right)|y|^{\frac{1}{2}}(1+|y|+\tau)^{-\frac{1}{2}+2 \mu}\left\{(1+|y|)^{-\frac{1}{2}}|v(y, \tau)|_{\kappa+3}+\right. \\
& \left.(1+|y|)^{-\frac{1}{2}}(1+|| y|-\bar{c} \tau|)|\partial v(y, \tau)|_{\kappa+2}+(1+|| y|-\bar{c} \tau|)(1+|y|+\tau)^{-\frac{1}{2}}|\partial v(y, \tau)|_{\kappa+2}\right\} \\
& \leq C\left([\partial v]_{\left[\frac{\kappa+2}{2}\right], t}+\langle v\rangle_{\left[\frac{\kappa+4}{2}\right], t}\right)|y|^{\frac{1}{2}}(1+|y|+\tau)^{-\frac{1}{2}+2 \mu}\left\{(1+|y|)^{-\frac{1}{2}}(1+|| y|-\bar{c} \tau|) \times\right. \\
& \left(|\nabla v(y, \tau)|_{\kappa+2}+(1+\tau)^{-1}|v(y, \tau)|_{\kappa+3}\right)+(1+|y|)^{-\frac{1}{2}}|v(y, \tau)|_{\kappa+3}+ \\
& \left.(1+|| y|-\bar{c} \tau|)(1+|y|+\tau)^{-\frac{1}{2}}\left(|\nabla v(y, \tau)|_{\kappa+2}+(1+\tau)^{-1}|v(y, \tau)|_{\kappa+3}\right)\right\} \\
& \leq C\left([\partial v]_{\left[\frac{\kappa+2}{2}\right], t}+\langle v\rangle_{\left[\frac{\kappa+4}{2}\right], t}\right)\left\{\varepsilon+|y|^{\frac{1}{2}}(1+|y|+\tau)^{-\frac{1}{2}+2 \mu}(1+|y|)^{-\frac{1}{2}}(1+|| y|-\bar{c} \tau|) \times\right. \\
& \left.|\nabla \Phi(F)(y, \tau)|_{\kappa+2}+|y|^{\frac{1}{2}}(1+|y|+\tau)^{-\frac{1}{2}+2 \mu}(1+|y|)^{-\frac{1}{2}}|\Phi(F)(y, \tau)|_{\kappa+3}\right\} \\
& \leq C\left([\partial v]_{\left[\frac{\kappa+2}{2}\right], t}+\langle v\rangle_{\left[\frac{\kappa+4}{2}\right], t}\right)\left\{\varepsilon+(1+|y|+\tau)^{-1+2 \mu}\left([\nabla \Phi(F)(y, \tau)]_{\kappa+2}+\langle\Phi(F)(y, \tau)\rangle_{\kappa+3}\right)\right\} \\
& \leq C\left([\partial v]_{\left[\frac{\kappa+2}{2}\right], t}+\langle v\rangle_{\left[\frac{\kappa+4}{2}\right], t}\right)\left(\varepsilon+[\partial v]_{\left[\frac{\kappa+4}{2}\right], t} \sup _{0 \leq \tau<t}\left\{(1+\tau)^{-\nu}\|\partial v(\tau)\|_{\kappa+6}\right\}\right) .
\end{aligned}
$$


Making use of (2.18), (4.5), (4.9) and (4.17) with $\theta=2$ gives

$$
\begin{aligned}
(1+|y|+ & \tau)^{\frac{1}{2}+2 \mu}|y|^{\frac{1}{2}}(1+|y|)\left|\sum_{i, j, k=0}^{2} g_{i j}^{k} \partial_{k} v \partial_{i} \partial_{j} v(y, \tau)\right|_{\kappa+1} \\
\leq & C|y|^{\frac{1}{2}}(1+|y|+\tau)^{\frac{1}{2}+2 \mu}(1+|y|)|\partial v(y, \tau)|_{\left[\frac{\kappa+2}{2}\right]}|\partial v(y, \tau)|_{\kappa+2} \\
\leq & C[\partial v]_{\left[\frac{\kappa+2}{2}\right], t}|y|^{\frac{1}{2}}(1+|y|+\tau)^{\frac{1}{2}+2 \mu}(1+|y|)^{\frac{1}{2}}(1+|| y|-\bar{c} \tau|)^{-1}|\partial v(y, \tau)|_{\kappa+2} \\
\leq & C[\partial v]_{\left[\frac{\kappa+2}{2}\right], t}|y|^{\frac{1}{2}}(1+|y|+\tau)^{\frac{1}{2}+2 \mu}(1+|y|)^{\frac{1}{2}}(1+|| y|-\bar{c} \tau|)^{-1} \times \\
& \left(|\nabla v(y, \tau)|_{\kappa+2}+(1+\tau)^{-1}|v(y, \tau)|_{\kappa+3}\right) \\
\leq & C[\partial v]_{\left[\frac{\kappa+2}{2}\right], t}\left\{\varepsilon+|y|^{\frac{1}{2}}(1+|y|+\tau)^{\frac{1}{2}+2 \mu}(1+\| y|-\bar{c} \tau|)^{-2}[\nabla \Phi(F)(y, \tau)]_{\kappa+2}+\right. \\
& \left.|y|^{\frac{1}{2}}(1+|y|+\tau)^{2 \mu}(1+|y|)^{-\frac{1}{2}}(1+|| y|-\bar{c} \tau|)^{-1}\langle\Phi(F)(y, \tau)\rangle_{\kappa+3}\right\} \\
\leq & C[\partial v]_{\left[\frac{\kappa+2}{2}\right], t}\left\{\varepsilon+(1+|y|+\tau)^{-1+2 \mu}\left([\nabla \Phi(F)(y, \tau)]_{\kappa+2}+\langle\Phi(F)(y, \tau)\rangle_{\kappa+3}\right)\right\} \\
\leq & C[\partial v]_{\left[\frac{\kappa+2}{2}\right], t}\left(\varepsilon+[\partial v]_{\left[\frac{\kappa+4}{2}\right], t} \sup _{0 \leq \tau<t}\left\{(1+\tau)^{-\nu}\|\partial v(\tau)\|_{\kappa+6}\right\}\right)
\end{aligned}
$$

for $(y, \tau) \in D(x, t) \bigcap \Lambda(t)^{c} \bigcap\{(z, s) \mid s \geq 1\}$. On the other hand, by (4.15), for $(y, \tau) \in$ $D(x, t) \bigcap \Lambda(t) \bigcap\{(z, s) \mid s \geq 1\}$ we have

$$
\begin{aligned}
(1+|y|+ & \tau)^{\frac{1}{2}+2 \mu}|y|^{\frac{1}{2}}(1+|| y|-\bar{c} \tau|)\left|\sum_{i, j, k, l=0}^{2} g_{i j}^{k l} \partial_{k} v \partial_{l} v \partial_{i} \partial_{j} v(y, \tau)\right|_{\kappa+1} \\
& \leq C[\partial v]_{\left[\frac{\kappa+2}{2}\right], t}^{2}|y|^{\frac{1}{2}}(1+|y|+\tau)^{\frac{1}{2}+2 \mu}(1+|| y|-\bar{c} \tau|)^{-1}(1+|y|)^{-1}|\partial v(y, \tau)|_{\kappa+2} \\
& \leq C[\partial v]_{\left[\frac{\kappa+2}{2}\right], t}^{2} \sup _{0 \leq \tau<t}\left\{(1+\tau)^{-\nu}\|\partial v(\tau)\|_{\kappa+4}\right\} .
\end{aligned}
$$

It follows from (2.18) and (4.15) that, for $(y, \tau) \in D(x, t) \bigcap \Lambda(t)^{c} \bigcap\{(z, s) \mid s \geq 1\}$

$$
\begin{aligned}
(1+|y|+ & \tau)^{\frac{1}{2}+2 \mu}|y|^{\frac{1}{2}}(1+|y|)\left|\sum_{i, j, k, l=0}^{2} g_{i j}^{k l} \partial_{k} v \partial_{l} v \partial_{i} \partial_{j} v(y, \tau)\right|_{\kappa+1} \\
& \leq C[\partial v]_{\left[\frac{\kappa+2}{2}\right], t}^{2}|y|^{\frac{1}{2}}(1+|y|+\tau)^{\frac{1}{2}+2 \mu}(1+|| y|-\bar{c} \tau|)^{-2}|\partial v(y, \tau)|_{\kappa+2} \\
& \leq C[\partial v]_{\left[\frac{\kappa+2}{2}\right], t}^{2} \sup _{0 \leq \tau<t}\left\{(1+\tau)^{-\nu}\|\partial v(\tau)\|_{\kappa+4}\right\}
\end{aligned}
$$

(4.22)-(4.28) yields (4.17) in the case $\theta=1$.

Case 3: $\theta=0$ 
For $(y, \tau) \in D(x, t) \bigcap \Lambda(t) \bigcap\{(z, s) \mid s \leq 1\}$, we obtain from (4.15) and (4.16) that

$$
\begin{aligned}
(1+|y|+ & \tau)^{1+\mu}|y|^{\frac{1}{2}}(1+|| y|-\bar{c} \tau|)\left(\left|\sum_{i, j, k=0}^{2} g_{i j}^{k} \partial_{k} v \partial_{i} \partial_{j} v(y, \tau)\right|_{\kappa+1}+\left|\sum_{i, j, k, l=0}^{2} g_{i j}^{k l} \partial_{k} v \partial_{l} v \partial_{i} \partial_{j} v(y, \tau)\right|_{\kappa+1}\right) \\
& \leq C[\partial v]_{\left[\frac{\kappa+2}{2}\right], t}|y|^{\frac{1}{2}}(1+|y|+\tau)^{1+\mu}(1+|y|)^{-\frac{1}{2}}|\partial v(y, \tau)|_{\kappa+2} \\
& \leq C[\partial v]_{\left[\frac{\kappa+2}{2}\right], t} \sup _{0 \leq \tau<t}\left\{(1+\tau)^{-\nu}\|\partial v(\tau)\|_{\kappa+4}\right\} .
\end{aligned}
$$

Similarly, it follows from (2.18), (4.15) and (4.16) that, for $(y, \tau) \in D(x, t) \bigcap \Lambda(t)^{c} \bigcap\{(z, s) \mid s \leq$ $1\}$

$$
\begin{aligned}
(1+|y|+ & \tau)^{1+\mu}|y|^{\frac{1}{2}}(1+|y|)\left(\left.\left|\sum_{i, j, k=0}^{2} g_{i j}^{k} \partial_{k} v \partial_{i} \partial_{j} v(y, \tau)\right|\right|_{\kappa+1}+\left|\sum_{i, j, k, l=0}^{2} g_{i j}^{k l} \partial_{k} v \partial_{l} v \partial_{i} \partial_{j} v(y, \tau)\right|_{\kappa+1}\right) \\
& \leq C[\partial v]_{\left[\frac{\kappa+2}{2}\right], t}|y|^{\frac{1}{2}}(1+|y|+\tau)^{1+\mu}(1+|y|)^{\frac{1}{2}}(1+|| y|-\bar{c} \tau|)^{-1}|\partial v(y, \tau)|_{\kappa+2} \\
& \leq C[\partial v]_{\left[\frac{\kappa+2}{2}\right], t} \sup _{0 \leq \tau<t}\left\{(1+\tau)^{-\nu}\|\partial v(\tau)\|_{\kappa+4}\right\} .
\end{aligned}
$$

We next consider the case $\tau \geq 1$. 
Noting (3.23)-(3.24), (4.5), (4.9), (4.16) and using (4.17) in the case $\theta=1$, for $(y, \tau) \in$ $D(x, t) \bigcap \Lambda(t) \bigcap\{(z, s) \mid s \geq 1\}$ we have

$$
\begin{aligned}
& (1+|y|+\tau)^{1+\mu}(1+|| y|-\bar{c} \tau|)|y|^{\frac{1}{2}}\left(\left|\sum_{i, j, k=0}^{2} g_{i j}^{k} \partial_{k} v \partial_{i} \partial_{j} v(y, \tau)\right|_{\kappa+1}+\left|\sum_{i, j, k, l=0}^{2} g_{i j}^{k l} \partial_{k} v \partial_{l} v \partial_{i} \partial_{j} v(y, \tau)\right|_{\kappa+1}\right) \\
& \leq C|y|^{\frac{1}{2}}(1+|y|+\tau)^{\mu}\left\{(1+|| y|-\bar{c} \tau|)^{2}|\partial v(y, \tau)|_{\left[\frac{\kappa+2}{2}\right]}|\partial v(y, \tau)|_{\kappa+2}+\right. \\
& \left.(1+|| y|-\bar{c} \tau|)\left(|\partial v(y, \tau)|_{\left[\frac{\kappa+2}{2}\right]}|v(y, \tau)|_{\kappa+3}+|v(y, \tau)|_{\left[\frac{\kappa+4}{2}\right]}|\partial v(y, \tau)|_{\kappa+2}\right)\right\} \\
& \leq C\left([\partial v]_{\left[\frac{\kappa+2}{2}\right], t}+\langle v\rangle_{\left[\frac{\kappa+4}{2}\right], t}\right)|y|^{\frac{1}{2}}(1+|y|+\tau)^{\mu}\left\{(1+|y|)^{-\frac{1}{2}}(1+|| y|-\bar{c} \tau|)|\partial v(y, \tau)|_{\kappa+2}+\right. \\
& \left.(1+|y|)^{-\frac{1}{2}}|v(y, \tau)|_{\kappa+3}+(1+|| y|-\bar{c} \tau|)(1+|y|+\tau)^{-\frac{1}{2}}|\partial v(y, \tau)|_{\kappa+2}\right\} \\
& \leq C\left([\partial v]_{\left[\frac{\kappa+2}{2}\right], t}+\langle v\rangle_{\left[\frac{\kappa+4}{2}\right], t}\right)|y|^{\frac{1}{2}}(1+|y|+\tau)^{\mu}\left\{(1+|y|)^{-\frac{1}{2}}(1+|| y|-\bar{c} \tau|) \times\right. \\
& \left(|\nabla v(y, \tau)|_{\kappa+2}+(1+\tau)^{-1}|v(y, \tau)|_{\kappa+3}\right)+(1+|y|)^{-\frac{1}{2}}|v(y, \tau)|_{\kappa+3}+ \\
& \left.(1+|| y|-\bar{c} \tau|)(1+|y|+\tau)^{-\frac{1}{2}}\left(|\nabla v(y, \tau)|_{\kappa+2}+(1+\tau)^{-1}|v(y, \tau)|_{\kappa+3}\right)\right\} \\
& \leq C\left([\partial v]_{\left[\frac{\kappa+2}{2}\right], t}+\langle v\rangle_{\left[\frac{\kappa+4}{2}\right], t}\right)\left\{\varepsilon+|y|^{\frac{1}{2}}(1+|y|+\tau)^{\mu}(1+|y|)^{-\frac{1}{2}}(1+|| y|-\bar{c} \tau|) \times\right. \\
& \left.|\nabla \Phi(F)(y, \tau)|_{\kappa+2}+|y|^{\frac{1}{2}}(1+|y|+\tau)^{\mu}(1+|y|)^{-\frac{1}{2}}|\Phi(F)(y, \tau)|_{\kappa+3}\right\} \\
& \leq C\left([\partial v]_{\left[\frac{\kappa+2}{2}\right], t}+\langle v\rangle_{\left[\frac{\kappa+4}{2}\right], t}\right)\left\{\varepsilon+(1+|y|+\tau)^{-\frac{1}{2}+\mu}\left([\nabla \Phi(F)(y, \tau)]_{\kappa+2}+\langle\Phi(F)(y, \tau)\rangle_{\kappa+3}\right)\right\} \\
& \leq C\left([\partial v]_{\left[\frac{\kappa+2}{2}\right], t}+\langle v\rangle_{\left[\frac{\kappa+4}{2}\right], t}\right)\left\{\varepsilon+\left([\partial v]_{\left[\frac{\kappa+6}{2}\right], t}+\langle v\rangle_{\left[\frac{\kappa+6}{2}\right], t}\right) \sup _{0 \leq \tau<t}\left\{(1+\tau)^{-\nu}\|\partial v(\tau)\|_{\kappa+8}\right\}\right\} .
\end{aligned}
$$


Using (2.18), (4.5), (4.9), (4.16) and noting (4.17) with $\theta=1$, for $(y, \tau) \in D(x, t) \bigcap \Lambda(t)^{c} \cap\{(z, s) \mid s \geq$ 1\} we obtain

$$
\begin{aligned}
(1+ & |y|+\tau)^{1+\mu}(1+|y|)|y|^{\frac{1}{2}}\left(\left|\sum_{i, j, k=0}^{2} g_{i j}^{k} \partial_{k} v \partial_{i} \partial_{j} v(y, \tau)\right|_{\kappa+1}+\left|\sum_{i, j, k, l=0}^{2} g_{i j}^{k l} \partial_{k} v \partial_{l} v \partial_{i} \partial_{j} v(y, \tau)\right|_{\kappa+1}\right) \\
& \leq C[\partial v]_{\left[\frac{\kappa+2}{2}\right], t}|y|^{\frac{1}{2}}(1+|y|+\tau)^{1+\mu}(1+|y|)^{\frac{1}{2}}(1+|| y|-\bar{c} \tau|)^{-1}|\partial v(y, \tau)|_{\kappa+2} \\
& \leq C[\partial v]_{\left[\frac{\kappa+2}{2}\right], t}|y|^{\frac{1}{2}}(1+|y|+\tau)^{1+\mu}(1+|y|)^{\frac{1}{2}}(1+|| y|-\bar{c} \tau|)^{-1}\left(|\nabla v(y, \tau)|_{\kappa+2}+(1+\tau)^{-1}|v(y, \tau)|_{\kappa+3}\right) \\
& \leq C[\partial v]_{\left[\frac{\kappa+2}{2}\right], t}\left\{\varepsilon+|y|^{\frac{1}{2}}(1+|y|+\tau)^{1+\mu}(1+|| y|-\bar{c} \tau|)^{-2}[\nabla \Phi(F)(y, \tau)]_{\kappa+2}+\right. \\
& \leq C[\partial v]_{\left[\frac{\kappa+2}{2}\right], t}\left\{\varepsilon+(1+|y|+\tau)^{-\frac{1}{2}+\mu}\left([\nabla \Phi(F)(y, \tau)]_{\kappa+2}+\langle\Phi(F)(y, \tau)\rangle_{\kappa+3}\right)\right\} \\
& \leq C[\partial v]_{\left[\frac{\kappa+2}{2}\right], t}\left\{\varepsilon+\left([\partial v]_{\left[\frac{\kappa+6}{2}\right], t}+\langle v\rangle_{\left[\frac{\kappa+6}{2}\right], t}\left(1+\mid y+\sup _{0 \leq \tau<t}\left\{(1+\tau)^{-\nu}\|\partial v(\tau)\|_{\kappa+8}\right\}\right)\right\}\right. \\
& \leq C\left([\partial v]_{\left[\frac{\kappa+6}{2}\right], t}+\langle v\rangle_{\left[\frac{\kappa+6}{2}\right], t}\right)\left(\varepsilon+\sup _{0 \leq \tau<t}\left\{(1+\tau)^{-\nu}\|\partial v(\tau)\|_{\kappa+8}\right\}\right) .
\end{aligned}
$$

Therefore, combining (4.11)-(4.12) and (4.29)-(4.32) gives (4.17) in the case $\theta=0$ immediately. Thus, the proof of Lemma 4.3 is completed.

\section{$5 \quad$ Energy estimate}

This section is devoted to establishing the energy estimates, which play an important role in the proof of Theorem 1.1.

Theorem 5.1 Let $v=v(x, t)$ be a smooth solution of the Cauchy problem (2.16)-(2.17) on the domain $\mathbb{R}^{2} \times[0, T)$ ). If there exists a positive small constant $\delta$ such that

$$
[\partial v]_{\left[\frac{\kappa+1}{2}\right], T}+\langle v\rangle_{\left[\frac{\kappa+1}{2}\right]+1, T} \leq \delta
$$

then, for every $t \in[0, T)$, it holds that

$$
\|\partial v(t)\|_{\kappa} \leq C_{2} \varepsilon(1+t) C^{C_{3}\left([\partial v]_{\left[\frac{\kappa+1}{2}\right], T}+\langle v\rangle_{\left[\frac{\kappa+1}{2}\right]+1, T}\right)},
$$

where $C_{2}$ and $C_{3}$ are two positive constants independent of $T$.

Proof. Define

$$
q(\lambda)=\int_{-\infty}^{\lambda} \frac{1}{(1+|\varrho|)^{2}} d \varrho
$$


and

$$
p(x, t)=q(|| x|-\bar{c} t|), \quad \dot{p}(x, t)=q^{\prime}(|x|-\bar{c} t) .
$$

Obviously,

$$
q \in C^{1}(\mathbb{R}), \quad p \in C^{1}\left(\mathbb{R}^{2} \times \mathbb{R}^{+}\right) \quad \text { and } \quad \dot{p} \in C^{0}\left(\mathbb{R}^{2} \times \mathbb{R}^{+}\right) .
$$

Moreover,

$$
0 \leq p(x, t) \leq 2
$$

and

$$
\dot{p}(x, t)=\frac{1}{(1+|| x|-\bar{c} t|)^{2}}
$$

for any $(x, t) \in \mathbb{R}^{2} \times(0,+\infty)$.

On the other hand, we introduce the following energy functions

$$
\begin{gathered}
E_{0}(v(t))=\left\{\int_{\mathbb{R}^{2}}\left(\left|\partial_{t} v(x, t)\right|^{2}+\bar{c}^{2}|\nabla v(x, t)|^{2}\right) d x\right\}^{\frac{1}{2}}, \\
E_{\kappa}(v(t))=\sum_{|a| \leq \kappa} E_{0}\left(\Gamma^{a} v(t)\right), \\
\tilde{E}_{0}(v(t))=\left\{\int_{\mathbb{R}^{2}} e^{p}(x, t)\left(\left|\partial_{t} v(x, t)\right|^{2}+\bar{c}^{2}|\nabla v(x, t)|^{2}\right) d x\right\}^{\frac{1}{2}}
\end{gathered}
$$

and

$$
\tilde{E}_{\kappa}(v(t))=\sum_{|a| \leq \kappa} \tilde{E}_{0}\left(\Gamma^{a} v(t)\right) .
$$

Thus, it follows from (5.4), (5.6)-(5.9) that

$$
\frac{1}{\hat{C}}\|\partial v(t)\|_{\kappa} \leq \frac{1}{\tilde{C}} E_{\kappa}(v(t)) \leq \tilde{E}_{\kappa}(v(t)) \leq \tilde{C} E_{\kappa}(v(t)) \leq \hat{C}\|\partial v(t)\|_{\kappa}
$$

for some positive constant $\hat{C}$ and $\tilde{C}$ which are independent of $t$. Therefore, in order to prove (5.2), it suffices to show

$$
\tilde{E}_{\kappa}(v(t)) \leq C \varepsilon(1+t) C\left([\partial v]_{\left[\frac{\kappa+1}{2}\right], T}+\langle v\rangle_{\left[\frac{\kappa+1}{2}\right]+1, T}\right)
$$

for $t \in[0, T)$. 
To do so, noting (2.16), we have

$$
\begin{aligned}
& \partial_{t}^{2} \Gamma^{a} v-\bar{c}^{2} \triangle \Gamma^{a} v+2 \sum_{i=1}^{2} \partial_{i} v \partial_{t} \partial_{i} \Gamma^{a} v-2 \partial_{t} v \triangle \Gamma^{a} v+\sum_{i, j=1}^{2} \partial_{i} v \partial_{j} v \partial_{i} \partial_{j} \Gamma^{a} v-|\nabla v|^{2} \triangle \Gamma^{a} v \\
&= \sum_{b \leq a} C_{b} \Gamma^{b}\left(-2 \sum_{i=1}^{2} \partial_{i} v \partial_{t} \partial_{i} v+2 \partial_{t} v \triangle v-\sum_{i, j=1}^{2} \partial_{i} v \partial_{j} v \partial_{i} \partial_{j} v+|\nabla v|^{2} \triangle v\right)+ \\
& 2 \sum_{i=1}^{2} \partial_{i} v \partial_{t} \partial_{i} \Gamma^{a} v-2 \partial_{t} v \triangle \Gamma^{a} v+\sum_{i, j=1}^{2} \partial_{i} v \partial_{j} v \partial_{i} \partial_{j} \Gamma^{a} v-|\nabla v|^{2} \triangle \Gamma^{a} v
\end{aligned}
$$

for any given multi-index $a$, where $C_{b}$ is a constant which is determined by (3.3). Notice that $C_{a}=1$. Multiplying $e^{p} \partial_{t} \Gamma^{a} v$ to (5.12) and then integrating with respect to $x$ on $\mathbb{R}^{2}$ leads to

$$
\begin{aligned}
\frac{1}{2} \frac{d}{d t} \int_{\mathbb{R}^{2}}\left\{e^{p}\left(\left|\partial_{t} \Gamma^{a} v(x, t)\right|^{2}+\bar{c}^{2}\left|\nabla \Gamma^{a} v(x, t)\right|^{2}\right)\right\} d x+ \\
\int_{\mathbb{R}^{2}} e^{p}\left(\frac{\bar{c}}{2} \dot{p}\left|Z \Gamma^{a} v\right|^{2}+2 \sum_{i=1}^{2} \partial_{i} v \partial_{t} \partial_{i} \Gamma^{a} v \partial_{t} \Gamma^{a} v-2 \partial_{t} v \triangle \Gamma^{a} v \partial_{t} \Gamma^{a} v+\right. \\
\left.\sum_{i, j=1}^{2} \partial_{i} v \partial_{j} v \partial_{i} \partial_{j} \Gamma^{a} v \partial_{t} \Gamma^{a} v-|\nabla v|^{2} \triangle \Gamma^{a} v \partial_{t} \Gamma^{a} v\right) d x=I_{1}(t),
\end{aligned}
$$

where

$$
\begin{aligned}
I_{1}(t)= & \int_{\mathbb{R}^{2}} e^{p} \partial_{t} \Gamma^{a} v\left\{\sum_{b \leq a} C_{b} \Gamma^{b}\left(-2 \sum_{i=1}^{2} \partial_{i} v \partial_{t} \partial_{i} v+2 \partial_{t} v \triangle v-\sum_{i, j=1}^{2} \partial_{i} v \partial_{j} v \partial_{i} \partial_{j} v+|\nabla v|^{2} \triangle v\right)+\right. \\
& \left.2 \sum_{i=1}^{2} \partial_{i} v \partial_{t} \partial_{i} \Gamma^{a} v-2 \partial_{t} v \triangle \Gamma^{a} v+\sum_{i, j=1}^{2} \partial_{i} v \partial_{j} v \partial_{i} \partial_{j} \Gamma^{a} v-|\nabla v|^{2} \triangle \Gamma^{a} v\right\} d x .
\end{aligned}
$$


Notice that

$$
\begin{aligned}
& e^{p}\left(2 \sum_{i=1}^{2} \partial_{i} v \partial_{t} \partial_{i} \Gamma^{a} v \partial_{t} \Gamma^{a} v-2 \partial_{t} v \triangle \Gamma^{a} v \partial_{t} \Gamma^{a} v+\sum_{i, j=1}^{2} \partial_{i} v \partial_{j} v \partial_{i} \partial_{j} \Gamma^{a} v \partial_{t} \Gamma^{a} v-|\nabla v|^{2} \triangle \Gamma^{a} v \partial_{t} \Gamma^{a} v\right) \\
& =\sum_{i=1}^{2} \partial_{i}\left(e^{p} \partial_{i} v \partial_{t} \Gamma^{a} v \partial_{t} \Gamma^{a} v\right)-2 \sum_{i=1}^{2} \partial_{i}\left(e^{p} \partial_{t} v \partial_{i} \Gamma^{a} v \partial_{t} \Gamma^{a} v\right)+\sum_{i=1}^{2} \partial_{t}\left(e^{p} \partial_{i} v \partial_{i} \Gamma^{a} v \partial_{i} \Gamma^{a} v\right)+ \\
& \sum_{i=1}^{2} \sum_{j=1}^{2} \partial_{i}\left(e^{p} \partial_{i} v \partial_{j} v \partial_{j} \Gamma^{a} v \partial_{t} \Gamma^{a} v\right)-\frac{1}{2} \sum_{i=1}^{2} \sum_{j=1}^{2} \partial_{t}\left(e^{p} \partial_{i} v \partial_{j} v \partial_{j} \Gamma^{a} v \partial_{i} \Gamma^{a} v\right)- \\
& \sum_{i=1}^{2} \partial_{i}\left(e^{p}|\nabla v|^{2} \partial_{i} \Gamma^{a} v \partial_{t} \Gamma^{a} v\right)+\frac{1}{2} \sum_{i=1}^{2} \partial_{t}\left(e^{p}|\nabla v|^{2} \partial_{i} \Gamma^{a} v \partial_{i} \Gamma^{a} v\right)- \\
& \frac{\dot{p}}{2} e^{p}\left(2 \sum_{i=1}^{2} \omega_{i} \partial_{i} v \partial_{t} \Gamma^{a} v \partial_{t} \Gamma^{a} v-4 \sum_{i=1}^{2} \omega_{i} \partial_{t} v \partial_{i} \Gamma^{a} v \partial_{t} \Gamma^{a} v-2 \bar{c} \sum_{i=1}^{2} \partial_{t} v \partial_{i} \Gamma^{a} v \partial_{i} \Gamma^{a} v+\right. \\
& 2 \sum_{i=1}^{2} \sum_{j=1}^{2} \omega_{i} \partial_{i} v \partial_{j} v \partial_{j} \Gamma^{a} v \partial_{t} \Gamma^{a} v+\bar{c} \sum_{i=1}^{2} \sum_{j=1}^{2} \partial_{i} v \partial_{j} v \partial_{j} \Gamma^{a} v \partial_{i} \Gamma^{a} v- \\
& \left.2 \sum_{i=1}^{2} \omega_{i}|\nabla v|^{2} \partial_{i} \Gamma^{a} v \partial_{t} \Gamma^{a} v-\bar{c} \sum_{i=1}^{2}|\nabla v|^{2} \partial_{i} \Gamma^{a} v \partial_{i} \Gamma^{a} v\right)- \\
& \frac{1}{2} e^{p}\left(2 \sum_{i=1}^{2} \partial_{i}^{2} v \partial_{t} \Gamma^{a} v \partial_{t} \Gamma^{a} v-4 \sum_{i=1}^{2} \partial_{i} \partial_{t} v \partial_{i} \Gamma^{a} v \partial_{t} \Gamma^{a} v+2 \sum_{i=1}^{2} \partial_{t}^{2} v \partial_{i} \Gamma^{a} v \partial_{i} \Gamma^{a} v+\right. \\
& 2 \sum_{i=1}^{2} \sum_{j=1}^{2} \partial_{i}\left(\partial_{i} v \partial_{j} v\right) \partial_{j} \Gamma^{a} v \partial_{t} \Gamma^{a} v-2 \sum_{i=1}^{2} \sum_{j=1}^{2} \partial_{t}\left(\partial_{i} v \partial_{j} v\right) \partial_{j} \Gamma^{a} v \partial_{i} \Gamma^{a} v- \\
& \left.2 \sum_{i=1}^{2} \partial_{i}\left(|\nabla v|^{2}\right) \partial_{i} \Gamma^{a} v \partial_{t} \Gamma^{a} v+\sum_{i=1}^{2} \partial_{t}\left(|\nabla v|^{2}\right) \partial_{i} \Gamma^{a} v \partial_{i} \Gamma^{a} v\right)
\end{aligned}
$$

where $\omega_{i}=\frac{x_{i}}{|x|}(i=1,2)$. It follows from (5.13) and (5.15) that

$$
\begin{gathered}
I_{1}(t)=\frac{1}{2} \frac{d}{d t} \int_{\mathbb{R}^{2}}\left\{e^{p}\left(\left|\partial_{t} \Gamma^{a} v(x, t)\right|^{2}+\bar{c}^{2}\left|\nabla \Gamma^{a} v(x, t)\right|^{2}\right)\right\} d x+\int_{\mathbb{R}^{2}} \frac{\bar{c}}{2} e^{p} \dot{p}\left|Z \Gamma^{a} v\right|^{2} d x+ \\
\frac{d}{d t} \int_{\mathbb{R}^{2}}\left(e^{p} \partial_{i} v \partial_{i} \Gamma^{a} v \partial_{i} \Gamma^{a} v-\frac{1}{2} \sum_{i=1}^{2} \sum_{j=1}^{2} e^{p} \partial_{i} v \partial_{j} v \partial_{j} \Gamma^{a} v \partial_{i} \Gamma^{a} v\right. \\
\left.+\frac{1}{2} \sum_{i=1} e^{p}|\nabla v|^{2} \partial_{i} \Gamma^{a} v \partial_{i} \Gamma^{a} v\right) d x+I_{2}(t)+I_{3}(t)
\end{gathered}
$$


where

$$
\begin{aligned}
I_{2}(t)=-\int_{\mathbb{R}^{2}} \frac{\dot{p}}{2} e^{p}( & 2 \sum_{i=1}^{2} \omega_{i} \partial_{i} v \partial_{t} \Gamma^{a} v \partial_{t} \Gamma^{a} v-4 \sum_{i=1}^{2} \omega_{i} \partial_{t} v \partial_{i} \Gamma^{a} v \partial_{t} \Gamma^{a} v-2 \bar{c} \sum_{i=1}^{2} \partial_{t} v \partial_{i} \Gamma^{a} v \partial_{i} \Gamma^{a} v+ \\
& 2 \sum_{i=1}^{2} \sum_{j=1}^{2} \omega_{i} \partial_{i} v \partial_{j} v \partial_{j} \Gamma^{a} v \partial_{t} \Gamma^{a} v+\bar{c} \sum_{i=1}^{2} \sum_{j=1}^{2} \partial_{i} v \partial_{j} v \partial_{j} \Gamma^{a} v \partial_{i} \Gamma^{a} v- \\
& \left.2 \sum_{i=1}^{2} \omega_{i}|\nabla v|^{2} \partial_{i} \Gamma^{a} v \partial_{t} \Gamma^{a} v-\bar{c} \sum_{i=1}^{2}|\nabla v|^{2} \partial_{i} \Gamma^{a} v \partial_{i} \Gamma^{a} v\right) d x
\end{aligned}
$$

and

$$
\begin{gathered}
I_{3}(t)=-\int_{\mathbb{R}^{2}} \frac{1}{2} e^{p}\left(2 \sum_{i=1}^{2} \partial_{i}^{2} v \partial_{t} \Gamma^{a} v \partial_{t} \Gamma^{a} v-4 \sum_{i=1}^{2} \partial_{i} \partial_{t} v \partial_{i} \Gamma^{a} v \partial_{t} \Gamma^{a} v+2 \sum_{i=1}^{2} \partial_{t}^{2} v \partial_{i} \Gamma^{a} v \partial_{i} \Gamma^{a} v+\right. \\
2 \sum_{i=1}^{2} \sum_{j=1}^{2} \partial_{i}\left(\partial_{i} v \partial_{j} v\right) \partial_{j} \Gamma^{a} v \partial_{t} \Gamma^{a} v-2 \sum_{i=1}^{2} \sum_{j=1}^{2} \partial_{t}\left(\partial_{i} v \partial_{j} v\right) \partial_{j} \Gamma^{a} v \partial_{i} \Gamma^{a} v- \\
\left.2 \sum_{i=1}^{2} \partial_{i}\left(|\nabla v|^{2}\right) \partial_{i} \Gamma^{a} v \partial_{t} \Gamma^{a} v+\sum_{i=1}^{2} \partial_{t}\left(|\nabla v|^{2}\right) \partial_{i} \Gamma^{a} v \partial_{i} \Gamma^{a} v\right) d x
\end{gathered}
$$

Combining (5.1) and (5.16) yields

$$
\tilde{E}_{\kappa}(v(t))^{2}+\sum_{|a| \leq \kappa} \int_{0}^{t} \int_{\mathbb{R}^{2}} e^{p} \dot{p}\left|Z \Gamma^{a} v(x, \tau)\right|^{2} d x d \tau \leq C \tilde{E}_{\kappa}(v(0))^{2}+C \int_{0}^{t}|I(\tau)| d \tau
$$

for $t \in[0, T)$, where

$$
I(t)=\sum_{|a| \leq \kappa}\left(\left|I_{1}(t)\right|+\left|I_{2}(t)\right|+\left|I_{3}(t)\right|\right) .
$$

Now we claim that

$$
\begin{aligned}
C|I(t)| \leq & \frac{1}{2} \sum_{|a| \leq \kappa} \int_{\mathbb{R}^{2}} \dot{p} e^{p}\left|Z \Gamma^{a} v(x, t)\right|^{2} d x+ \\
& \frac{C}{1+t}\left([\partial v]_{\left[\frac{\kappa+1}{2}\right], T}+\langle v\rangle_{\left[\frac{\kappa+1}{2}\right]+1, T}\right) \int_{\mathbb{R}^{2}} e^{p}|\partial v(x, t)|_{\kappa}^{2} d x
\end{aligned}
$$

for $t \in[0, T)$.

For the time being, we assume that (5.21) is true. We will prove it later. Thus, it follows from (5.19) and (5.21) that

$$
\begin{aligned}
& \tilde{E}_{\kappa}(v(t))^{2}+\frac{1}{2} \sum_{|a| \leq \kappa} \int_{0}^{t} \int_{\mathbb{R}^{2}} e^{p} \dot{p}\left|Z \Gamma^{a} v(x, \tau)\right|^{2} d x d \tau \\
& \leq C \tilde{E}_{\kappa}(v(0))^{2}+C \int_{0}^{t} \frac{1}{1+\tau}\left([\partial v]_{\left[\frac{\kappa+1}{2}\right], T}+\langle v\rangle_{\left[\frac{\kappa+1}{2}\right]+1, T}\right) \tilde{E}_{\kappa}(v(\tau))^{2} d \tau .
\end{aligned}
$$


Noting (2.17), (5.22) and using Gronwall inequality gives (5.11) immediately.

It suffices to prove (5.21) in the rest of this section. To prove (5.21), we distinguish two cases.

Case I: $t \in[0,1]$

In this case, (5.21) comes from (5.14), (5.17) and (5.18) immediately.

\section{Case II: $t \in[1, T)$}

We first estimate $\left|I_{1}(t)\right|$.

Noting (2.18), (2.20), (2.21), (3.7), (3.20), (3.21), (5.1), (5.14) and using Cauchy inequality, we have

$$
\begin{aligned}
& \left|I_{1}(t)\right| \leq \int_{(x, t) \in \Lambda(T)}\left|\Gamma^{a}\left(\sum_{i, j, k=0}^{2} g_{i j}^{k} \partial_{k} v \partial_{i} \partial_{j} v\right)-\sum_{i, j, k=0}^{2} g_{i j}^{k} \partial_{k} v \partial_{i} \partial_{j} \Gamma^{a} v\right|\left|\partial_{t} \Gamma^{a} v\right| e^{p} d x+ \\
& \sum_{b<a} \int_{(x, t) \in \Lambda(T)}\left|C_{b} \Gamma^{b}\left(\sum_{i, j, k=0}^{2} g_{i j}^{k} \partial_{k} v \partial_{i} \partial_{j} v\right)\right|\left|\partial_{t} \Gamma^{a} v\right| e^{p} d x+ \\
& \sum_{b \leq a} \int_{(x, t) \in \Lambda(T)^{c}}\left|C_{b} \Gamma^{b}\left(\sum_{i, j, k=0}^{2} g_{i j}^{k} \partial_{k} v \partial_{i} \partial_{j} v\right)-\sum_{i, j, k=0}^{2} g_{i j}^{k} \partial_{k} v \partial_{i} \partial_{j} \Gamma^{a} v\right|\left|\partial_{t} \Gamma^{a} v\right| e^{p} d x+ \\
& \sum_{b \leq a} \int_{\mathbb{R}^{2}}\left|C_{b} \Gamma^{b}\left(\sum_{i, j, k, l=0}^{2} g_{i j}^{k l} \partial_{k} v \partial_{l} v \partial_{i} \partial_{j} v\right)-\sum_{i, j, k, l=0}^{2} g_{i j}^{k l} \partial_{k} v \partial_{l} v \partial_{i} \partial_{j} \Gamma^{a} v\right|\left|\partial_{t} \Gamma^{a} v\right| e^{p} d x \\
& \leq C \int_{(x, t) \in \Lambda(T)}\left(\frac{|| x|-\bar{c} t|}{1+|x|+t}|\partial v|_{\left[\frac{\kappa+1}{2}\right]}+\frac{1}{1+|x|+t}|v|_{\left[\frac{\kappa+1}{2}\right]+1}\right) e^{p}|\partial v|_{\kappa}^{2} d x+ \\
& C \sum_{|b| \leq \kappa} \int_{(x, t) \in \Lambda(T)} e^{p}|\partial v|_{\left[\frac{\kappa+1}{2}\right]}\left|Z \Gamma^{b} v\right||\partial v|_{\kappa} d x+C \int_{(x, t) \in \Lambda(T)^{c}} e^{p}|\partial v|_{\left[\frac{\kappa+1}{2}\right]}|\partial v|_{\kappa}^{2} d x+ \\
& C \int_{\mathbb{R}^{2}} e^{p}|\partial v|_{\left[\frac{\kappa+1}{2}\right]}^{2}|\partial v|_{\kappa}^{2} d x \\
& \leq C \int_{(x, t) \in \Lambda(T)}\left(\frac{|| x|-\bar{c} t|}{1+|x|+t}|\partial v|_{\left[\frac{\kappa+1}{2}\right]}+\frac{1}{1+|x|+t}|v|_{\left[\frac{\kappa+1}{2}\right]+1}\right) e^{p}|\partial v|_{\kappa}^{2} d x+ \\
& C \sum_{|b| \leq \kappa} \int_{(x, t) \in \Lambda(T)} e^{p}|\partial v|_{\left[\frac{\kappa+1}{2}\right]}\left|Z \Gamma^{b} v \| \partial v\right|_{\kappa} d x+ \\
& \int_{\mathbb{R}^{2}} e^{p}\left(\frac{1}{1+t}[\partial v]_{\left[\frac{\kappa+1}{2}\right], T}+|\partial v|_{\left[\frac{\kappa+1}{2}\right]}^{2}\right)|\partial v|_{\kappa}^{2} d x \\
& \leq \epsilon \sum_{|b| \leq \kappa} \int_{\mathbb{R}^{2}} \frac{e^{p}}{(1+|| x|-\bar{c} t|)^{2}}\left|Z \Gamma^{b} v\right|^{2} d x+\frac{C_{\epsilon}}{1+t}\left([\partial v]_{\left[\frac{\kappa+1}{2}\right], T}+\langle v\rangle_{\left[\frac{\kappa+1}{2}\right]+1, T}\right) \int_{\mathbb{R}^{2}} e^{p}|\partial v(x, t)|_{\kappa}^{2} d x \\
& \leq \epsilon \sum_{|b| \leq \kappa} \int_{\mathbb{R}^{2}} \dot{p} e^{p}\left|Z \Gamma^{b} v\right|^{2} d x+\frac{C_{\epsilon}}{1+t}\left([\partial v]_{\left[\frac{\kappa+1}{2}\right], T}+\langle v\rangle_{\left[\frac{\kappa+1}{2}\right]+1, T}\right) \int_{\mathbb{R}^{2}} e^{p}|\partial v(x, t)|_{\kappa}^{2} d x,
\end{aligned}
$$


where $\epsilon$ is a positive small constant.

We now estimate $\left|I_{2}(t)\right|$.

Noting (2.18), (3.7), (5.1), (5.17) and using the null condition and Cauchy inequality, we obtain

$$
\begin{aligned}
& \left|I_{2}(t)\right| \leq \int_{(x, t) \in \Lambda(T)} \dot{p} e^{p} \mid \sum_{i=1}^{2} \omega_{i} \partial_{i} v \partial_{t} \Gamma^{a} v \partial_{t} \Gamma^{a} v-2 \sum_{i=1}^{2} \omega_{i} \partial_{t} v \partial_{i} \Gamma^{a} v \partial_{t} \Gamma^{a} v- \\
& \bar{c} \sum_{i=1}^{2} \partial_{t} v \partial_{i} \Gamma^{a} v \partial_{i} \Gamma^{a} v\left|d x+\int_{(x, t) \in \Lambda(T)^{c}} \dot{p} e^{p}\right| \sum_{i=1}^{2} \omega_{i} \partial_{i} v \partial_{t} \Gamma^{a} v \partial_{t} \Gamma^{a} v- \\
& 2 \sum_{i=1}^{2} \omega_{i} \partial_{t} v \partial_{i} \Gamma^{a} v \partial_{t} \Gamma^{a} v-\bar{c} \sum_{i=1}^{2} \partial_{t} v \partial_{i} \Gamma^{a} v \partial_{i} \Gamma^{a} v \mid d x+ \\
& \int_{\mathbb{R}^{2}} \frac{\dot{p}}{2} e^{p} \mid 2 \sum_{i=1}^{2} \sum_{j=1}^{2} \omega_{i} \partial_{i} v \partial_{j} v \partial_{j} \Gamma^{a} v \partial_{t} \Gamma^{a} v+\bar{c} \sum_{i=1}^{2} \sum_{j=1}^{2} \partial_{i} v \partial_{j} v \partial_{j} \Gamma^{a} v \partial_{i} \Gamma^{a} v- \\
& 2 \sum_{i=1}^{2} \omega_{i}|\nabla v|^{2} \partial_{i} \Gamma^{a} v \partial_{t} \Gamma^{a} v-\bar{c} \sum_{i=1}^{2}|\nabla v|^{2} \partial_{i} \Gamma^{a} v \partial_{i} \Gamma^{a} v \mid d x \\
& \leq \int_{(x, t) \in \Lambda(T)} \dot{p} e^{p} \mid \sum_{i=1}^{2} \omega_{i} \partial_{i} v \partial_{t} \Gamma^{a} v \partial_{t} \Gamma^{a} v-2 \sum_{i=1}^{2} \omega_{i} \partial_{t} v \partial_{i} \Gamma^{a} v \partial_{t} \Gamma^{a} v- \\
& \bar{c} \sum_{i=1}^{2} \partial_{t} v \partial_{i} \Gamma^{a} v \partial_{i} \Gamma^{a} v \mid d x+C\left(\left.\int_{(x, t) \in \Lambda(T)^{c}}|\partial v \|| \partial v\right|_{\kappa} ^{2} d x+\int_{\mathbb{R}^{2}}|\partial v|^{2}|\partial v|_{\kappa}^{2} d x\right) \\
& \leq \int_{(x, t) \in \Lambda(T)} \dot{p} e^{p} \mid \frac{\omega_{1}}{\bar{c}}\left(\bar{c} \partial_{1}+\omega_{1} \partial_{t}\right) v\left(\partial_{t} \Gamma^{a} v\right)^{2}+\frac{\omega_{2}}{\bar{c}}\left(\bar{c} \partial_{2}+\omega_{2} \partial_{t}\right) v\left(\partial_{t} \Gamma^{a} v\right)^{2}- \\
& \frac{1}{\bar{c}} \partial_{t} v\left(\bar{c} \partial_{1}+\omega_{1} \partial_{t}\right) \Gamma^{a} v\left(\bar{c} \partial_{1}+\omega_{1} \partial_{t}\right) \Gamma^{a} v-\frac{1}{\bar{c}} \partial_{t} v\left(\bar{c} \partial_{2}+\omega_{2} \partial_{t}\right) \Gamma^{a} v\left(\bar{c} \partial_{2}+\omega_{2} \partial_{t}\right) \Gamma^{a} v- \\
& \frac{\omega_{1}}{\bar{c}} \partial_{t} v\left(\bar{c} \partial_{1}+\omega_{1} \partial_{t}\right) \Gamma^{a} v \partial_{t} \Gamma^{a} v-\frac{\omega_{2}}{\bar{c}} \partial_{t} v\left(\bar{c} \partial_{2}+\omega_{2} \partial_{t}\right) \Gamma^{a} v \partial_{t} \Gamma^{a} v \mid d x+ \\
& \frac{C}{1+t}[\partial v]_{0, T} \int_{\mathbb{R}^{2}} e^{p}|\partial v|_{\kappa}^{2} d x \\
& \leq C \int_{(x, t) \in \Lambda(T)} \dot{p} e^{p}\left(\left.\left|Z v\left\|\left.\partial \Gamma^{a} v\right|^{2}+\left|\partial v\left\|Z \Gamma^{a} v\right\| \partial \Gamma^{a} v\right|\right) d x+\frac{C}{1+t}[\partial v]_{0, T} \int_{\mathbb{R}^{2}} e^{p}\right| \partial v(x, t)\right|_{\kappa} ^{2} d x\right. \\
& \leq \epsilon \sum_{|a| \leq \kappa} \int_{\mathbb{R}^{2}} \dot{p} e^{p}\left|Z \Gamma^{a} v(x, t)\right|^{2} d x+\frac{C_{\epsilon}}{1+t}\left([\partial v]_{0, T}+\langle v\rangle_{1, T}\right) \int_{\mathbb{R}^{2}} e^{p}|\partial v(x, t)|_{\kappa}^{2} d x .
\end{aligned}
$$

where $\omega_{i}=\frac{x_{i}}{|x|}(i=1,2)$ and $\epsilon$ is a positive small constant.

Similarly, using (2.18), (3.7), (5.1), (5.18), the null condition and Cauchy inequality, we have

$$
\left|I_{3}(t)\right| \leq \epsilon \sum_{|a| \leq \kappa} \int_{\mathbb{R}^{2}} \dot{p} e^{p}\left|Z \Gamma^{a} v(x, t)\right|^{2} d x+\frac{C_{\epsilon}}{1+t}[\partial v]_{1, T} \int_{\mathbb{R}^{2}} e^{p}|\partial v(x, t)|_{\kappa}^{2} d x .
$$


Choosing $\epsilon$ suitably small, by (5.23)-(5.25) we see that (5.21) holds for $t \in[1, T)$. Thus the proof of Theorem 5.1 is completed.

\section{Proof of Theorem 1.1}

This section is devoted to the proof of Theorem 1.1. In order to prove Theorem 1.1, it suffices to show the following theorem.

Theorem 6.1 Under the assumptions of Theorem 1.1, there exists a positive constant $\varepsilon_{0}$ such that, for every $\varepsilon \in\left[0, \varepsilon_{0}\right]$, the smooth solution $v=v(x, t)$ of the Cauchy problem (2.16)-(2.17) exists globally in time.

Proof. The local existence of classical solutions has been proved by the method of Picard iteration (see Sogge [19] and Hörmander [9]). It suffices to show that if $v=v(x, t)$ is a smooth solution of the Cauchy problem (2.16)-(2.17) on the domain $\mathbb{R}^{2} \times[0, T)$ ), then $\sum_{|\alpha| \leq 4}\left|\partial^{\alpha} v(x, t)\right| \in L^{\infty}\left(\mathbb{R}^{2} \times[0, T)\right)$.

In what follows, we prove Theorem 6.1 by the method of continuous induction, or say, by the bootstrap argument.

Taking an integer $\kappa \geq 8$, we have

$$
\left[\frac{\kappa+9}{2}\right] \leq \kappa
$$

According to the bootstrap argument, we first assume that

$$
[\partial v]_{\kappa, T}+\langle v\rangle_{\kappa+1, T} \leq M \varepsilon
$$

and then we prove that, by choosing $M$ sufficiently large and $\varepsilon$ suitably small we have

$$
[\partial v]_{\kappa, T}+\langle v\rangle_{\kappa+1, T} \leq \frac{1}{2} M \varepsilon .
$$

To do so, let $\delta$ be a positive and small constant. Choosing $\varepsilon_{1} \in\left[0, \frac{\delta}{M}\right]$, we have

$$
\begin{aligned}
{[\partial v]_{\left[\frac{\kappa+6}{2}\right], T}+\langle v\rangle_{\left[\frac{\kappa+6}{2}\right], T} } & \leq[\partial v]_{\left[\frac{\kappa+9}{2}\right], T}+\langle v\rangle_{\left[\frac{\kappa+9}{2}\right]+1, T} \\
& \leq[\partial v]_{\kappa, T}+\langle v\rangle_{\kappa+1, T} \leq M \varepsilon \leq \delta
\end{aligned}
$$


for $\varepsilon \in\left[0, \frac{\delta}{M}\right]$. Thus, it follows from Theorems 4.1 and 5.1 that

$$
\begin{aligned}
{[\partial v]_{\kappa, T}+\langle v\rangle_{\kappa+1, T} } & \leq C_{0} \varepsilon+C_{1}\left([\partial v]_{\left[\frac{\kappa+6}{2}\right], T}+\langle v\rangle_{\left[\frac{\kappa+6}{2}\right], T}\right) \sup _{0 \leq \tau<T}\left\{(1+\tau)^{-\nu}\|\partial v(\tau)\|_{\kappa+8}\right\} \\
& \leq C_{0} \varepsilon+C_{1} C_{2} M \varepsilon^{2} \sup _{0 \leq \tau<T}\left\{(1+\tau)^{C_{3} M \varepsilon-\nu}\right\} \\
& \leq 2 C_{0} \varepsilon
\end{aligned}
$$

for $\varepsilon \in\left[0, \varepsilon_{2}\right]$, provided that

$$
\varepsilon_{2} \in\left[0, \min \left\{\frac{C_{0}}{C_{1} C_{2} M}, \frac{\nu}{C_{3} M}\right\}\right]
$$

where $\nu$ is the constant appearing in Theorem 4.1. Therefore, taking $\varepsilon_{0}=\min \left\{\varepsilon_{1}, \varepsilon_{2}\right\}$ and $M \geq 4 C_{0}$, we obtain (6.2) from (6.4) immediately. This implies that

$$
\sum_{|\alpha| \leq 4}\left|\partial^{\alpha} v(x, t)\right| \in L^{\infty}\left(\mathbb{R}^{2} \times[0, T)\right)
$$

Thus, the proof of Theorem 6.1 is completed.

Acknowledgements. This work was supported by the NNSF of China (Grant No. 10671124) and the Qiu-Shi Chair Professor Fellowship from Zhejiang University.

\section{References}

[1] S. Alinhac, The null condition for quasilinear wave equations in two space dimensions I, Invent. Math. 145 (2001), 597-618.

[2] M. Bordeman and J. Hoppe, The dynamics of relativistic membranes: Reduction to 2-dimensional fluid dynamics, Phys. Lett. B 317 (1993), 315-320.

[3] D. Christodoulou, Global solutions of nonlinear hyperbolic equations for small initial data, Comm. Pure Appl. Math. 39 (1986), 267-282.

[4] R. Courant and K. O. Friedrichs, Supersonic Flow and Shock Waves, Appl. Math. Sci. 21, Spring-Verlag, 1976.

[5] R. T. Glassey, Existence in the large for $\square u=F(u)$ in two space dimensions, Math. Z. 178 (1981), 233-261. 
[6] P. Godin, The lifespan of a class of smooth spherically symmetric solutions of the compressible Euler equations with variable entropy in three space dimensions, Arch. Rat. Mech. Anal. 177 (2005), 479-511.

[7] P. Godin, Global existence of a class of smooth 3D spherically symmetric flows of Chaplygin gases with variable entropy, J. Math. Pure Appl. 87 (2007), 91-117.

[8] M. Grassin, Global smooth solutions to Euler equations for perfect gas, Indiana. Univ. Math. J. 47 (1998), 1397-1432.

[9] L. Hörmander, Lectures on Nonlinear Hyperbolic Differential Equations, Mathématique and Applications 26, Spring-Verlag, 1997.

[10] A. Hoshiga, Existence and blowing up of solutions to systems of quasilinear wave equations in two space dimensions, Adv. Math. Sci. Appl. 15 (2005), 69-110.

[11] A. Hoshiga, The existence of global solutions to systems of quasilinear wave equations with quadratic nonlinearities in 2-dimensional space, Funkcialaj Ekvacioj 69 (2006), 357-384.

[12] S.-J. Huang and D.-X. Kong, Equations for the motion of relativistic torus in the Minkowski space $\mathbb{R}^{1+n}$, J. Math. Phys. 48 (2007), 103508-1-103508-15.

[13] S. Klainerman, Uniform decay estimates and the lorentz invariance of the classical wave equations, Comm. Pure Appl. Math. 38 (1985), 321-332.

[14] S. Klainerman, The null condition and global existence to nonlinear wave equations, Lectures in Appl. Math. 23, Amer. Math. Soc., 1986, 293-326.

[15] S. Klainerman, Remarks on the global Sobolev inequalities in the Minkowski space $\mathbb{R}^{n+1}$, Comm. Pure Appl. Math. 40 (1987), 111-116.

[16] M. Kovalyov, Long-time bahaviour of solutions of systems of nonlinear wave equations, Comm. Partial Differential Equations 12 (1987), 471-501.

[17] T. C. Sideris, Formation of singularities in three-dimensional compressible fluids, Comm. Math. Phys. 101 (1985), 475-485.

[18] T. C. Sideris, Delayed singularity formation in 2D compressible flow, Amer. J. Math. 119 (1997), 371-422. 
[19] C. D. Sogge, Lectures on Nonlinear Wave Equations, Monographs in Analysis II, International Press, 1995.

[20] C. Rogers and W. F. Shadwick, Bäcklund Transformations and Their Applications, Academic Press, New York, London, 1982.

[21] B. L. Roždestvenski and N. N. Janenko, Systems of Quasilinear Equations and Their Applications to Gas Dynamics, Translations of Mathematical Monographs 55, Amer. Math. Soc., Providence, RI, 1983. 